TITLE:

\title{
Ground-state phase diagram of the one-dimensional half-filled extended Hubbard model
}

\section{$\operatorname{AUTHOR}(\mathrm{S}):$}

Tsuchiizu, M; Furusaki, A

\section{CITATION:}

Tsuchiizu, M ...[et al]. Ground-state phase diagram of the one-

dimensional half-filled extended Hubbard model. PHYSICAL REVIEW B 2004, 69(3): 035103.

\section{ISSUE DATE:}

2004-01

URL:

http://hdl.handle.net/2433/50485

RIGHT:

Copyright 2004 American Physical Society 
PHYSICAL REVIEW B 69, 035103 (2004)

\title{
Ground-state phase diagram of the one-dimensional half-filled extended Hubbard model
}

\author{
M. Tsuchiizu ${ }^{1,2}$ and A. Furusaki ${ }^{1,3}$ \\ ${ }^{1}$ Yukawa Institute for Theoretical Physics, Kyoto University, Kyoto 606-8502, Japan \\ ${ }^{2}$ Department of Physics, Nagoya University, Nagoya 464-8602, Japan \\ ${ }^{3}$ Condensed-Matter Theory Laboratory, The Institute of Physical and Chemical Research (RIKEN), Saitama 351-0198, Japan
}

(Received 7 August 2003; published 15 January 2004)

\begin{abstract}
We revisit the ground-state phase diagram of the one-dimensional half-filled extended Hubbard model with on-site $(U)$ and nearest-neighbor $(V)$ repulsive interactions. In the first half of the paper, using the weakcoupling renormalization-group approach ( $g$-ology) including second-order corrections to the coupling constants, we show that bond-charge-density-wave (BCDW) phase exists for $U \approx 2 V$ in between charge-densitywave $(\mathrm{CDW})$ and spin-density-wave (SDW) phases. We find that the umklapp scattering of parallel-spin electrons disfavors the BCDW state and leads to a bicritical point where the CDW-BCDW and SDW-BCDW continuous-transition lines merge into the CDW-SDW first-order transition line. In the second half of the paper, we investigate the phase diagram of the extended Hubbard model with either additional staggered site potential $\Delta$ or bond alternation $\delta$. Although the alternating site potential $\Delta$ strongly favors the CDW state (that is, a band insulator), the BCDW state is not destroyed completely and occupies a finite region in the phase diagram. Our result is a natural generalization of the work by Fabrizio, Gogolin, and Nersesyan [Phys. Rev. Lett. 83, 2014 (1999)], who predicted the existence of a spontaneously dimerized insulating state between a band insulator and a Mott insulator in the phase diagram of the ionic Hubbard model. The bond alternation $\delta$ destroys the SDW state and changes it into the BCDW state (or Peierls insulating state). As a result the phase diagram of the model with $\delta$ contains only a single critical line separating the Peierls insulator phase and the CDW phase. The addition of $\Delta$ or $\delta$ changes the universality class of the CDW-BCDW transition from the Gaussian transition into the Ising transition.
\end{abstract}

DOI: 10.1103/PhysRevB.69.035103

PACS number(s): 71.10.Fd, 71.10.Hf, 71.10.Pm, 71.30.+h

\section{INTRODUCTION}

It is well known that a one-dimensional (1D) spin system has instability to dimerization that changes the system into a nonmagnetic insulating state, the so-called spin-Peierls state. ${ }^{1}$ Indeed the spin-Peierls state is realized in many systems including quasi-one-dimensional organic compounds ${ }^{2,3}$ and the inorganic material ${ }^{4} \mathrm{CuGeO}_{3}$, and its properties have been studied extensively both experimentally and theoretically. Of particular interest is a situation in which a dimerized state appears spontaneously due to strong correlations and frustration. ${ }^{5}$ A well-known example is the frustrated spin- $\frac{1}{2}$ Heisenberg chain with nearest-neighbor, $J_{1}$, and nextnearest-neighbor, $J_{2}$, antiferromagnetic exchange interactions, where a spontaneously dimerized phase is realized for $J_{2} \geqslant J_{2 c} \simeq 0.24 J_{1}{ }^{6}$ Other systems of current interest are quasi-one-dimensional electron systems in organic materials, where the spin-Peierls state appears due to strong electron correlation at half filling ${ }^{7-14}$ and at quarter filling. ${ }^{15,16}$

Recently it was pointed out by Nakamura and co-workers ${ }^{17}$ that a spontaneously dimerized state occupies a finite parameter space in the ground-state phase diagram of the 1D half-filled Hubbard model with the nearest-neighbor repulsion $V$, i.e., the extended Hubbard model (EHM). This spin-Peierls state is often called bond-charge-density-wave (BCDW) state or bond-ordered-wave state. The appearance of the BCDW state in the purely electronic model is nontrivial and has attracted much attention from theoretical point of view. To appreciate this surprising result, let us consider some limiting cases. In the limit of weak nearest-neighbor repulsion $V$, or in the half-filled Hubbard model with only the on-site Coulomb repulsion $U$, the ground state is in the Mott insulating state where the spin sector exhibits quasilong-range order of spin-density wave (SDW); we call it the SDW state. In the opposite limit of strong $V$, the ground state of the half-filled EHM has a long-range order of the chargedensity wave (CDW); we call this state the CDW state. Furthermore, in the atomic limit where the electron hopping $t$ is ignored, the CDW state appears for $U<2 V$ whereas the uniform state corresponding to the SDW state is stable for $U$ $>2 \mathrm{~V}$ in one dimension. Strong-coupling perturbation theory in $t$ has established that a first-order phase transition between the SDW state and the CDW state occurs at $U \simeq 2 V .{ }^{18-21}$ As for the weak-coupling regime, perturbative renormalizationgroup (RG) approach or $g$-ology led to a similar conclusion that the ground state at half filling is either in the SDW state or in the CDW state with a continuous phase-transition line at $U=2 V .{ }^{18}$ Thus, it had been considered for a long time that the ground-state phase diagram of the EHM at half filling has only two phases, the SDW and CDW states, and that the order of the phase transition at $U \simeq 2 V$ changes from continuous to first order at a tricritical point which was speculated to exist in the intermediate coupling regime. $^{20,22-24}$ This common view was revised by the Nakamura's discovery that the BCDW state exists at $U \simeq 2 \mathrm{~V}$ in between the SDW and CDW phases in the weak-coupling region, ${ }^{17}$ which is supported by recent large-scale Monte Carlo calculations. ${ }^{25,26}$ Related studies of the dimerized state in the EHM with additional correlation effects can be found in Refs. 27-31.

A related and still controversial issue of current interest is whether or not a spontaneously dimerized phase exists in the 
1D Hubbard model with alternating site potential, the socalled ionic Hubbard model. ${ }^{32-45}$ This system was introduced as a simple minimal model for the neutral-ionic transitions observed in quasi-one-dimensional organic materials $^{46-48}$ and for ferroelectric perovskites. ${ }^{49,50}$ Obviously the model has two insulating phases. The ground state is (i) a band insulator with the CDW order when the staggered site potential is much larger than the on-site repulsion or (ii) a Mott insulator with quasi-long-range SDW order when the staggered site potential is negligible. Early exact diagonalization studies ${ }^{49-51}$ of small systems have found a transition between the two phases and also reported dramatic enhancement of the electron-lattice interaction by strong electron correlation near a boundary between the band insulating phase (the BI state) and the Mott insulating phase (the SDW state). Mostly through bosonization analysis of the ionic Hubbard model, Fabrizio, Gogolin, and Nersesyan recently argued ${ }^{32}$ that a phase of a spontaneously dimerized insulator (SDI) intervenes between the ionic insulating phase (band insulator) and the Mott insulating phase. The SDI state is closely related to the BCDW state mentioned above. Earlier numerical studies ${ }^{34-36,38,39,51}$ have drawn contradictory conclusions as to whether the SDI phase exists or not, but more recent numerical studies find two phase transitions and the SDI phase in between. ${ }^{37,40,45}$ Nevertheless there still remain unresolved issues on the critical properties near the quantum phase transitions.

In this paper we give supporting theoretical arguments for the existence of the spontaneously dimerized insulating states in the 1D half-filled extended Hubbard model with and without staggered potentials. We adopt the standard bosonization approach and perform both perturbative RG analysis valid in the weak-coupling regime and semiclassical analysis which is expected to give a qualitatively correct picture even in the strong-coupling regime. This paper is organized as follows. Sections II and III are devoted to the analysis of the standard EHM, i.e., the system without the staggered potential. Some of the results of this part are already presented in Ref. 52. In Sec. II, we introduce the model and reformulate the weak-coupling theory, the $g$-ology, to include higher-order corrections to coupling constants. We bosonize low-energy effective Hamiltonian and derive the renormalization-group equations. In Sec. III, we determine the ground-state phase diagram. First, from the perturbative RG analysis we show that the BCDW phase occupies a finite region near the $U=2 V$ line in the weakcoupling limit. Next, from the semiclassical analysis we argue that the umklapp scattering of parallel-spin electrons destabilizes the BCDW phase and gives rise to a bicritical point where the CDW-BCDW and SDW-BCDW continuoustransition lines merge into the CDW-SDW first-order transition line. Finally, combining the perturbative RG equations with the semiclassical analysis, we obtain the global phase diagram of the 1D EHM. In Sec. IV we study the 1D EHM with the staggered site potential. We take the same strategy as in the previous sections and perform a semiclassical analysis of the bosonized Hamiltonian. With the help of the perturbative RG analysis we obtain the global phase diagram that indeed has the SDI phase. We find that the BCDW phase of the EHM is continuously deformed to the SDI phase upon introducing the alternating site potential. In Sec. V, we study the 1D EHM with additional bond dimerization, but without the staggered potential. This model exhibits a quantum phase transition between a dimerized Peierls insulator and a CDW state. Section VI is devoted to conclusions, and details of the technical calculations are given in Appendixes.

\section{EXTENDED HUBBARD MODEL}

In the first half of this paper (Secs. II and III), we consider the standard 1D EHM which has on-site, $U$, and nearestneighbor, $V$, interactions. The Hamiltonian is given by

$$
\begin{aligned}
H= & -t \sum_{j, \sigma}\left(c_{j, \sigma}^{\dagger} c_{j+1, \sigma}+\text { H.c. }\right)+U \sum_{j} n_{j, \uparrow} n_{j, \downarrow} \\
& +V \sum_{j} n_{j} n_{j+1},
\end{aligned}
$$

where $n_{j, \sigma} \equiv c_{j, \sigma}^{\dagger} c_{j, \sigma}-\frac{1}{2}, n_{j} \equiv n_{j, \uparrow}+n_{j, \downarrow}$, and $c_{j, \sigma}^{\dagger}$ denotes the creation operator of an electron with spin $\sigma(=\uparrow, \downarrow)$ on the $j$ th site. We assume repulsive interactions, i.e., the coupling constants $U$ and $V$ are positive. Note that the Hamiltonian has global $\mathrm{SU}(2)$ spin symmetry. Following the previous studies on models with correlated-hopping interactions, ${ }^{28}$ we consider the CDW, SDW, BCDW, and bond-spin-density-wave (BSDW) phases as potential ordered ground states at half filling. They are characterized by the order parameters

$$
\begin{gathered}
\mathcal{O}_{\mathrm{CDW}} \equiv(-1)^{j}\left(n_{j, \uparrow}+n_{j, \downarrow}\right), \\
\mathcal{O}_{\mathrm{SDW}} \equiv(-1)^{j}\left(n_{j, \uparrow}-n_{j, \downarrow}\right), \\
\mathcal{O}_{\mathrm{BCDW}} \equiv(-1)^{j}\left(c_{j, \uparrow}^{\dagger} c_{j+1, \uparrow}+c_{j, \downarrow}^{\dagger} c_{j+1, \downarrow}+\text { H.c. }\right), \\
\mathcal{O}_{\mathrm{BSDW}} \equiv(-1)^{j}\left(c_{j, \uparrow}^{\dagger} c_{j+1, \uparrow}-c_{j, \downarrow}^{\dagger} c_{j+1, \downarrow}+\text { H.c. }\right) .
\end{gathered}
$$

The order parameter of the BCDW state corresponds to the Peierls dimerization operator. We note that the BCDW state can be also regarded as the $p$-density-wave state, ${ }^{53}$ as the order parameter of the BCDW state can be written as $\quad \sum_{j} \mathcal{O}_{\mathrm{BCDW}} \propto \sum_{k, \sigma} \sin (k a) c_{k, \sigma}^{\dagger} c_{k+(\pi / a), \sigma}$, where $c_{k, \sigma}$ $=N^{-1 / 2} \sum_{j} e^{-i k R_{j}} c_{j, \sigma}$ with $R_{j}=j a$ ( $a$ : the lattice spacing, $N$ : the number of sites). The BSDW state describes a site-offdiagonal SDW state. ${ }^{28}$

\section{A. g-ology approach}

The hopping $t$ generates the energy band with dispersion $\varepsilon_{k}=-2 t \cos k a$, where the Fermi points are at $k= \pm k_{F}$ $= \pm \pi / 2 a$ at half filling. In order to analyze the low-energy physics near the Fermi points, we introduce a momentum cutoff $\Lambda\left(0<\Lambda<k_{F}\right)$ and divide the momentum space into the three sectors (Fig. 1) (i) $k \in R$, (ii) $k \in L$, and (iii) $k \notin(R \cup L)$, where $R=\left[k_{F}-\Lambda, k_{F}+\Lambda\right]$ and $L=\left[-k_{F}-\Lambda\right.$, $\left.-k_{F}+\Lambda\right]$. We then introduce the following fermion operators: 


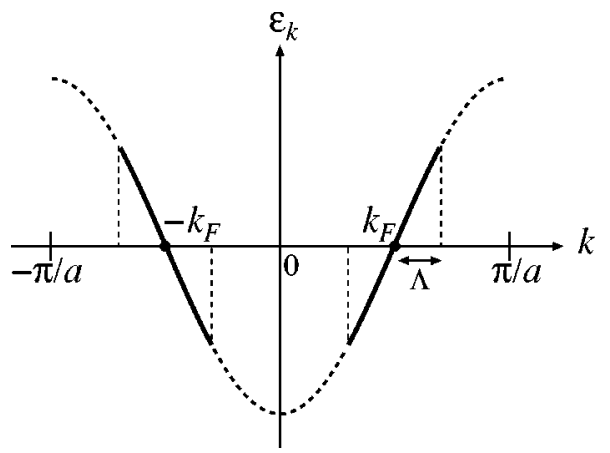

FIG. 1. Single-particle energy band. The annihilation operator of an electron near the Fermi points with momentum $k \in\left[-k_{F}-\Lambda\right.$, $\left.-k_{F}+\Lambda\right]\left(k \in\left[k_{F}-\Lambda, k_{F}+\Lambda\right]\right)$ is denoted $a_{k,-, \sigma}\left(a_{k,+, \sigma}\right)$, and that of an electron far away from the Fermi points is denoted $b_{k, \sigma}$.

$$
c_{k, \sigma}= \begin{cases}a_{k,+, \sigma} & \text { for } k \in R \\ a_{k,-, \sigma} & \text { for } k \in L \\ b_{k, \sigma} & \text { otherwise. }\end{cases}
$$

Electrons near the Fermi points are shuffled by the twoparticle scattering: $H_{\text {int }}=U \Sigma_{j} n_{j, \uparrow} n_{j, \downarrow}+V \Sigma_{j} n_{j} n_{j+1}$. Following the standard $g$-ology approach, ${ }^{18,54}$ we will focus on the scattering processes between electrons near the Fermi points, i.e., the scattering processes which involve $a_{k, \pm, \sigma}$ only. The Hamiltonian for such interaction processes is

$$
\begin{aligned}
H_{\mathrm{int}}= & +\frac{g_{1 \|}}{2 L} \sum_{k_{i}, p, \sigma}: a_{k_{1}, p, \sigma}^{\dagger} a_{k_{2},-p, \sigma} a_{k_{3},-p, \sigma}^{\dagger} a_{k_{4}, p, \sigma}: \\
& +\frac{g_{1 \perp}}{2 L} \sum_{k_{i}, p, \sigma}: a_{k_{1}, p, \sigma}^{\dagger} a_{k_{2},-p, \sigma} a_{k_{3},-p, \bar{\sigma}}^{\dagger} a_{k_{4}, p, \bar{\sigma}}: \\
& +\frac{g_{2 \|}}{2 L} \sum_{k_{i}, p, \sigma}: a_{k_{1}, p, \sigma}^{\dagger} a_{k_{2}, p, \sigma} a_{k_{3},-p, \sigma}^{\dagger} a_{k_{4},-p, \sigma}: \\
& +\frac{g_{2 \perp}}{2 L} \sum_{k_{i}, p, \sigma}: a_{k_{1}, p, \sigma}^{\dagger} a_{k_{2}, p, \sigma} a_{k_{3},-p, \bar{\sigma}}^{\dagger} a_{k_{4},-p, \bar{\sigma}}: \\
& +\frac{g_{3 \|}}{2 L} \sum_{k_{i}, p, \sigma}: a_{k_{1}, p, \sigma}^{\dagger} a_{k_{2},-p, \sigma} a_{k_{3}, p, \sigma}^{\dagger} a_{k_{4},-p, \sigma}: \\
& +\frac{g_{3 \perp}}{2 L} \sum_{k_{i}, p, \sigma}: a_{k_{1}, p, \sigma}^{\dagger} a_{k_{2},-p, \sigma} a_{k_{3}, p, \bar{\sigma}}^{\dagger} a_{k_{4},-p, \bar{\sigma}}: \\
& +\frac{g_{4 \|}}{2 L} \sum_{k_{i}, p, \sigma}: a_{k_{1}, p, \sigma}^{\dagger} a_{k_{2}, p, \sigma} a_{k_{3}, p, \sigma}^{\dagger} a_{k_{4}, p, \sigma}: \\
& +\frac{g_{4 \perp}}{2 L} \sum_{k_{i}, p, \sigma}: a_{k_{1}, p, \sigma}^{\dagger} a_{k_{2}, p, \sigma} a_{k_{3}, p, \sigma}^{\dagger} a_{k_{4}, p, \bar{\sigma}}:,
\end{aligned}
$$

where $\bar{\sigma}=\downarrow(\uparrow)$ for $\sigma=\uparrow(\downarrow), L$ is the length of the system, and : : denotes normal ordering. The summation over the momentum $k_{i}$ is taken under the condition of the total momentum being conserved (equal to $\pm 2 \pi / a$ for the umklapp scattering). The index $p=+/-$ denotes the right-/leftmoving electron. The coupling constants $g_{1 \|}$ and $g_{1 \perp}\left(g_{3 \|}\right.$ and $g_{3 \perp}$ ) denote the matrix elements of the backward (um- klapp) scattering, while $g_{2 \|}$ and $g_{2 \perp}\left(g_{4 \|}\right.$ and $\left.g_{4 \perp}\right)$ denote the matrix element of the forward scattering with the different (same) branch $p= \pm$. The index $\|(\perp)$ of the coupling constants denotes the scattering of electrons with same (opposite) spins.

\section{B. Vertex corrections}

In the conventional weak-coupling approach to the 1D EHM, ${ }^{17,18}$ one estimates the coupling constants in Eq. (2.4) only up to the lowest order in $U$ and $V$ :

$$
\begin{gathered}
g_{1 \perp}=g_{3 \perp}=(U-2 V) a, \\
g_{2 \perp}=g_{4 \perp}=(U+2 V) a, \\
g_{1 \|}=g_{3 \|}=-2 V a, \\
g_{2 \|}=g_{4 \|}=+2 V a .
\end{gathered}
$$

In analyzing the low-energy physics of Eq. (2.4), one then employs the standard $g$-ology approach, ${ }^{54}$ i.e., the perturbative RG method, and obtains flow equations for the marginal terms in Eq. (2.4). From this RG analysis ${ }^{18,54}$ one finds that the $g_{3 \perp}$ term generates a gap in the charge excitation spectrum if $\left|g_{3 \perp}\right|>-\left(g_{2 \|}+g_{2 \perp}-g_{1 \|}\right)$ and $g_{3 \perp} \neq 0$, whereas the $g_{1 \perp}$ term yields a gap in the spin excitation spectrum if $\left|g_{1 \perp}\right|>-\left(g_{2 \|}-g_{2 \perp}-g_{1 \|}\right)$ and $g_{1 \perp} \neq 0$. Hence, with the lowest-order coupling constants Eq. (2.5), one would conclude that the charge (spin) excitations become massless at $U-2 V=0 \quad(U-2 V \geqslant 0)$. This would mean that, as $U$ increases, both the charge and spin sectors become critical simultaneously at $U=2 \mathrm{~V}$, where a direct and continuous CDW-SDW transition takes place. This analysis is found to be insufficient from the following argument. The (accidental) simultaneous vanishing of $g_{3 \perp}$ and $g_{1 \perp}$ results from the lowest-order estimate in $U$ and $V$ and there is no symmetry principle that enforces $g_{1 \perp}$ and $g_{3 \perp}$ to vanish simultaneously. It is possible that the higher-order corrections to $g$ lift the degeneracy of zeros and change the topology of the phase diagram. Therefore, in order to analyze the phase diagram at $U \approx 2 V$, we need to go beyond the lowest-order calculation of the coupling constants in the g-ology. In this section, we compute the vertex corrections due to virtual processes involving high-energy states $^{55}$ by integrating out $b_{k, \sigma}$. This procedure allows us to obtain the effective coupling constants $g$ 's that include higher-order corrections.

The second-order vertex diagrams for the coupling constants are shown in Fig. 2. The solid lines denote the lowenergy states $a_{k, \pm, \sigma}$, while the dashed lines denote highenergy states $b_{k, \sigma}$. The nonzero contributions from the second-order virtual processes (a)-(e) are

$$
\begin{gathered}
\delta g_{1 \perp}^{(a)}=-\delta g_{3 \perp}^{(b)}=-\frac{U^{2}}{4 \pi t} D_{1} a+\frac{V^{2}}{\pi t} D_{2} a, \\
\delta g_{1 \perp}^{(c)}=+\delta g_{3 \perp}^{(c)}=+\frac{V(U-2 V)}{\pi t} D_{1} a,
\end{gathered}
$$



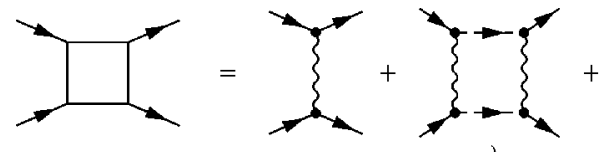

a)
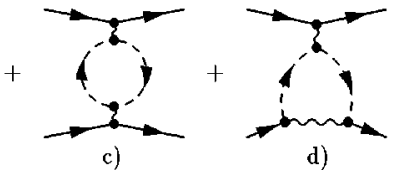

d)

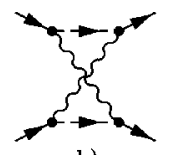

b)

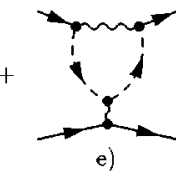

FIG. 2. Vertex diagrams with second-order corrections [(a)-(e)]. Solid lines denote electron states in the momentum space $k \in R$ or $k \in L$, while the dashed lines denote electron states in the other momentum space.

$$
\begin{gathered}
\delta g_{2 \perp}^{(a)}=-\delta g_{2 \perp}^{(b)}=-\frac{U^{2}}{4 \pi t} D_{1} a-\frac{V^{2}}{\pi t} D_{2} a, \\
\delta g_{1 \|}^{(a)}=+\frac{V^{2}}{\pi t} D_{2} a, \\
\delta g_{1 \|}^{(c)}=-\frac{(U-2 V)^{2}+4 V^{2}}{4 \pi t} D_{1} a-\frac{V^{2}}{\pi t} D_{2} a, \\
\delta g_{2 \|}^{(a)}=-\frac{V^{2}}{\pi t} D_{2} a, \\
\delta g_{3 \|}^{(c)}=-\frac{(U-2 V)^{2}+4 V^{2}}{4 \pi t} D_{1} a+\frac{V^{2}}{\pi t} D_{2} a,
\end{gathered}
$$

where

$$
\begin{gathered}
D_{1}(\Lambda) \equiv \int_{-\pi / 2+a \Lambda}^{\pi / 2-a \Lambda} \frac{d k}{\cos k}, \\
D_{2}(\Lambda) \equiv \int_{-\pi / 2+a \Lambda}^{\pi / 2-a \Lambda} d k \frac{\sin ^{2} k}{\cos k} .
\end{gathered}
$$

By introducing $C_{1}(\Lambda) \equiv 2 \ln [\cot (a \Lambda / 2)] \quad$ and $\quad C_{2}(\Lambda)$ $\equiv 2 \cos a \Lambda, D_{1}(\Lambda)$ and $D_{2}(\Lambda)$ are rewritten as $D_{1}(\Lambda)$ $=C_{1}(\Lambda)$ and $D_{2}(\Lambda)=C_{1}(\Lambda)-C_{2}(\Lambda)$. In terms of $C_{1}$ and $\mathrm{C}_{2}$, the coupling constants with second-order corrections are given by

$$
\begin{gathered}
g_{1 \perp}=(U-2 V) a\left[1-\frac{C_{1}}{4 \pi t}(U-2 V)\right]-\frac{C_{2}}{\pi t} V^{2} a, \\
g_{1 \|}=-2 V a-\frac{C_{1}}{4 \pi t}(U-2 V)^{2} a-\frac{C_{2}}{\pi t} V^{2} a, \\
g_{3 \perp}=(U-2 V) a\left[1+\frac{C_{1}}{4 \pi t}(U+6 V)\right]+\frac{C_{2}}{\pi t} V^{2} a, \\
g_{3 \|}=-2 V a-\frac{C_{1}}{4 \pi t}(U-2 V)^{2} a+\frac{C_{2}}{\pi t} V^{2} a,
\end{gathered}
$$

and $g_{2 \|}=+2 V a, g_{2 \perp}=(U+2 V) a, g_{4 \|}=+2 V a$, and $g_{4 \perp}$ $=(U+2 V) a$. Except when $a \Lambda \ll 1$, the $C_{i}$ 's depend on $\Lambda$ only weakly, and we can set $\Lambda=\pi / 4$ in the following analysis as we are interested in the qualitative feature of the phase diagram (different choices will only lead to small quantitative changes in phase boundaries). Incidentally, the logarithmic divergence of $C_{1}(\Lambda)$ in the limit $\Lambda \rightarrow 0$ leads to the familiar one-loop RG equations.

\section{Bosonization}

Having integrated out the high-energy virtual scattering processes, we now focus on the low-energy states and linearize the dispersion of $a_{k, \pm, \sigma}$ around the Fermi points. The kinetic-energy term with the linearized dispersion is given by

$$
\begin{aligned}
H_{0}= & \sum_{k \in R, \sigma} v_{F}\left(k-k_{F}\right) a_{k,+, \sigma}^{\dagger} a_{k,+, \sigma} \\
& +\sum_{k \in L, \sigma} v_{F}\left(-k-k_{F}\right) a_{k,-, \sigma}^{\dagger} a_{k,-, \sigma},
\end{aligned}
$$

where $v_{F}=2 t a$ is the Fermi velocity. The field operators of the right- and left-moving electrons are given by

$$
\begin{aligned}
& \psi_{+, \sigma}(x) \equiv \frac{1}{\sqrt{L}} \sum_{k \in R} e^{i k x} a_{k,+, \sigma}, \\
& \psi_{-, \sigma}(x) \equiv \frac{1}{\sqrt{L}} \sum_{k \in L} e^{i k x} a_{k,-, \sigma} .
\end{aligned}
$$

We apply the Abelian bosonization method and rewrite the kinetic-energy term $H_{0}=\int d x \mathcal{H}_{0}$ in terms of bosonic phase fields as (see Appendix A)

$$
\mathcal{H}_{0}=\frac{v_{F}}{4 \pi}\left[\left(2 \pi \Pi_{\theta}\right)^{2}+\left(\partial_{x} \theta\right)^{2}\right]+\frac{v_{F}}{4 \pi}\left[\left(2 \pi \Pi_{\phi}\right)^{2}+\left(\partial_{x} \phi\right)^{2}\right],
$$

where $\theta(\phi)$ is the bosonic field whose spatial derivative is proportional to the charge (spin) density, $[\theta(x), \phi(y)]=0$. The operators $\Pi_{\theta}$ and $\Pi_{\phi}$ are canonically conjugate variables to $\theta$ and $\phi$, respectively, and satisfy the conventional commutation relations, $\left[\theta(x), \Pi_{\theta}\left(x^{\prime}\right)\right]=\left[\phi(x), \Pi_{\phi}\left(x^{\prime}\right)\right]$ $=i \delta\left(x-x^{\prime}\right)$. We also introduce chiral bosonic fields

$$
\begin{gathered}
\theta_{ \pm}(x) \equiv \frac{1}{2}\left[\theta(x) \mp 2 \pi \int_{-\infty}^{x} d x^{\prime} \Pi_{\theta}\left(x^{\prime}\right)\right], \\
\phi_{ \pm}(x) \equiv \frac{1}{2}\left[\phi(x) \mp 2 \pi \int_{-\infty}^{x} d x^{\prime} \Pi_{\phi}\left(x^{\prime}\right)\right] .
\end{gathered}
$$

One can easily verify that these chiral fields satisfy the commutation relations $\left[\theta_{ \pm}(x), \theta_{ \pm}\left(x^{\prime}\right)\right]=\left[\phi_{ \pm}(x), \phi_{ \pm}\left(x^{\prime}\right)\right]$ $= \pm i(\pi / 2) \operatorname{sgn}\left(x-x^{\prime}\right) \quad$ and $\quad\left[\theta_{+}(x), \theta_{-}\left(x^{\prime}\right)\right]$ $=\left[\phi_{+}(x), \phi_{-}\left(x^{\prime}\right)\right]=i \pi / 2$. In terms of these fields, the kinetic-energy density reads 


$$
\mathcal{H}_{0}=\frac{v_{F}}{2 \pi} \sum_{p=+,-}\left[\left(\partial_{x} \theta_{p}\right)^{2}+\left(\partial_{x} \phi_{p}\right)^{2}\right]
$$

To express the electron field operators $\psi_{p, \sigma}$ with the bosonic phase fields, we introduce a new set of chiral bosonic fields

$$
\varphi_{p, \uparrow}=\theta_{p}+\phi_{p}, \quad \varphi_{p, \downarrow}=\theta_{p}-\phi_{p},
$$

which obey the commutation relations

$$
\begin{gathered}
{\left[\varphi_{ \pm, \sigma}(x), \varphi_{ \pm, \sigma^{\prime}}\left(x^{\prime}\right)\right]= \pm i \pi \operatorname{sgn}\left(x-x^{\prime}\right) \delta_{\sigma, \sigma^{\prime}},} \\
{\left[\varphi_{+, \sigma}(x), \varphi_{-, \sigma^{\prime}}\left(x^{\prime}\right)\right]=i \pi \delta_{\sigma, \sigma^{\prime}} .}
\end{gathered}
$$

In terms of the phase fields $\varphi_{p, \sigma}$, the electron field operators can be written as

$$
\psi_{p, \sigma}(x)=\frac{\eta_{\sigma}}{\sqrt{2 \pi a}} \exp \left[i p k_{F} x+i p \varphi_{p, \sigma}(x)\right],
$$

where the Klein factor $\eta_{\sigma}$ satisfies the anticommutation relation $\left\{\eta_{\sigma}, \eta_{\sigma^{\prime}}\right\}=2 \delta_{\sigma, \sigma^{\prime}}$. One can verify that the operator defined in Eq. (2.17) satisfies the same anticommutation relation as the fermion field operator. It follows from Eq. (2.17) that the order parameters in Eq. (2.2) are rewritten as

$$
\begin{gathered}
\mathcal{O}_{\mathrm{SDW}}(x) \propto \cos \theta(x) \sin \phi(x), \\
\mathcal{O}_{\mathrm{CDW}}(x) \propto \sin \theta(x) \cos \phi(x), \\
\mathcal{O}_{\mathrm{BCDW}}(x) \propto \cos \theta(x) \cos \phi(x), \\
\mathcal{O}_{\mathrm{BSDW}}(x) \propto \sin \theta(x) \sin \phi(x) .
\end{gathered}
$$

The interaction part of the Hamiltonian $H_{\text {int }}$, Eq. (2.4), can be also expressed in terms of the boson fields $\theta_{ \pm}$and $\phi_{ \pm}$. It has been suggested that, besides the marginal operators, operators with higher scaling dimensions can play an important role in the first-order CDW-SDW transition ${ }^{22,24}$ which is known to occur in the strong-coupling region of the 1D EHM. ${ }^{18-21}$ We thus include all the terms of scaling dimension $4[=2$ (charge sector) +2 ( spin sector) $]$. We also note that there are some complications and subtleties in bosonizing the off-site interaction term, i.e., the nearestneighbor interaction term $V$ (see Appendix A for detail). We obtain the bosonized Hamiltonian density,

$$
\begin{aligned}
\mathcal{H}= & \frac{1}{2 \pi} \sum_{p=+,-}\left[v_{\rho}\left(\partial_{x} \theta_{p}\right)^{2}+v_{\sigma}\left(\partial_{x} \phi_{p}\right)^{2}\right] \\
& +\frac{g_{\rho}}{2 \pi^{2}}\left(\partial_{x} \theta_{+}\right)\left(\partial_{x} \theta_{-}\right)-\frac{g_{\sigma}}{2 \pi^{2}}\left(\partial_{x} \phi_{+}\right)\left(\partial_{x} \phi_{-}\right) \\
& -\frac{g_{c}}{2 \pi^{2} a^{2}} \cos 2 \theta+\frac{g_{s}}{2 \pi^{2} a^{2}} \cos 2 \phi \\
& -\frac{g_{c s}}{2 \pi^{2} a^{2}} \cos 2 \theta \cos 2 \phi
\end{aligned}
$$

$$
\begin{aligned}
& -\frac{g_{\rho s}}{2 \pi^{2}}\left(\partial_{x} \theta_{+}\right)\left(\partial_{x} \theta_{-}\right) \cos 2 \phi \\
& +\frac{g_{c \sigma}}{2 \pi^{2}}\left(\partial_{x} \phi_{+}\right)\left(\partial_{x} \phi_{-}\right) \cos 2 \theta \\
& +\frac{g_{\rho \sigma}}{2 \pi^{2}} a^{2}\left(\partial_{x} \theta_{+}\right)\left(\partial_{x} \theta_{-}\right)\left(\partial_{x} \phi_{+}\right)\left(\partial_{x} \phi_{-}\right) .
\end{aligned}
$$

The renormalized velocities are $v_{\rho}=2 t a+\left(g_{4 \|}+g_{4 \perp}\right.$ $\left.-g_{1 \|}\right) / 2 \pi$ and $v_{\sigma}=2 t a+\left(g_{4 \|}-g_{4 \perp}-g_{1 \|}\right) / 2 \pi$. The marginal terms with the couplings $g_{\rho}$ and $g_{c}\left(g_{\sigma}\right.$ and $\left.g_{s}\right)$ determine low-energy properties of the charge (spin) modes, ${ }^{18,54}$ where $g_{\rho}=g_{2 \perp}+g_{2 \|}-g_{1 \|}, g_{c}=g_{3 \perp}, g_{\sigma}=g_{2 \perp}-g_{2 \|}+g_{1 \|}$, and $g_{s}=g_{1 \perp}$. The $g_{c s}, g_{\rho s}, g_{c \sigma}$, and $g_{\rho \sigma}$ terms with scaling dimension 4 couple the spin and charge degrees of freedom. The $g_{c s}$ coupling comes from the umklapp scattering $g_{3 \|}$. The $g_{\rho s}\left(g_{\rho \sigma}\right)$ coupling is generated from the backward scattering of antiparallel- (parallel-) spin electrons while the $g_{c \sigma}$ coupling is generated from the umklapp scattering of electrons with antiparallel spins (see Appendix A). These coupling constants are given by $g_{c s}=g_{\rho s}=g_{c \sigma}=g_{\rho \sigma}=-2 \mathrm{Va}$ to lowest order in $V$. Cannon and Fradkin examined the effect of the $g_{c s}$ term and argued that it plays a crucial role in the first-order CDW-SDW transition. ${ }^{22}$ Voit included the $g_{\rho s}$ and $g_{c \sigma}$ terms, as well as the $g_{c s}$ term, in the perturbative RG analysis of the coupling constants, but did not consider the $g_{\rho \sigma}$ term. ${ }^{24}$ Here we note that it is important to keep the $g_{\rho \sigma}$ term as well, since the global SU(2) symmetry in the spin sector is guaranteed only when $g_{\sigma}=g_{s}, g_{c s}=g_{c \sigma}$, and $g_{\rho s}$ $=g_{\rho \sigma}$.

\section{Renormalization-group equations}

We perform a perturbative RG calculation to examine the low-energy properties of the 1D EHM in the weak-coupling regime, taking into account quantum fluctuations of the phase fields. The operator product expansion (OPE) technique allows us to systematically handle the higher-order terms in the bosonized Hamiltonian (2.19). The one-loop RG equations that describe changes in the coupling constants during the scaling of the short-distance cutoff $\left(a \rightarrow a e^{d l}\right)$ are given by (see Appendix B for their derivation)

$$
\begin{gathered}
\frac{d}{d l} G_{\rho}=+2 G_{c}^{2}+G_{c s}^{2}+G_{s} G_{\rho s} \\
\frac{d}{d l} G_{c}=+2 G_{\rho} G_{c}-G_{s} G_{c s}-G_{c s} G_{\rho s}, \\
\frac{d}{d l} G_{s}=-2 G_{s}^{2}-G_{c} G_{c s}-G_{c s}^{2}, \\
\frac{d}{d l} G_{c s}=-2 G_{c s}+2 G_{\rho} G_{c s}-4 G_{s} G_{c s}-2 G_{c} G_{s} \\
-2 G_{c} G_{\rho s}-4 G_{c s} G_{\rho s},
\end{gathered}
$$




$$
\frac{d}{d l} G_{\rho s}=-2 G_{\rho s}+2 G_{\rho} G_{s}-4 G_{c} G_{c s}-4 G_{c s}^{2}-4 G_{s} G_{\rho s},
$$

where $G_{\nu}$ are dimensionless coupling constants with the initial values $G_{\nu}(0)=g_{\nu} /(4 \pi t a)$. The number of the independent coupling constants is five, since the $\mathrm{SU}(2)$ spin symmetry guarantees the relations $G_{\sigma}=G_{s}, G_{c \sigma}=G_{c s}$, and $G_{\rho \sigma}$ $=G_{\rho s}$ to hold in the scaling procedure. From these scaling equations, one finds that the $G_{\rho}, G_{c}$, and $G_{s}$ terms are marginal (the scaling dimension=2), ${ }^{56,57}$ while the $G_{c s}$ and $G_{\rho s}$ terms are irrelevant operators of the scaling dimension 4.

\section{PHASE DIAGRAM OF THE HALF-FILLED EXTENDED HUBBARD MODEL}

\section{A. Bond-charge-density-wave state}

In this section, we show that the BCDW phase exists in between the CDW and SDW phases in the weak-coupling region of the $1 \mathrm{D}$ EHM.

First we focus on the weak-coupling limit $U, V \ll t$, where we can neglect the irrelevant terms of scaling dimension 4 and restrict ourselves to the marginal terms $\propto g_{\rho}, g_{\sigma}, g_{c}$, and $g_{s}$. Effects of the irrelevant terms are discussed later in this section. Within this approximation, the Hamiltonian reduces to two decoupled sine-Gordon models, and we can analyze the properties of the spin and charge modes, separately. The one-loop RG equations for these coupling constants are given by Eqs. (2.20)-(2.22) with $G_{c s}=G_{\rho s}=0$ :

$$
\begin{gathered}
\frac{d}{d l} G_{\rho}(l)=2 G_{c}^{2}(l), \\
\frac{d}{d l} G_{c}(l)=2 G_{\rho}(l) G_{c}(l), \\
\frac{d}{d l} G_{s}(l)=-2 G_{s}^{2}(l) .
\end{gathered}
$$

The spin excitations are controlled by the $G_{s}$ coupling, which is marginally relevant (marginally irrelevant) when $G_{s}<0\left(G_{s}>0\right)$. If $g_{s}<0$, then $\left|G_{s}(l)\right|$ increases with increasing $l$. In this case the phase field $\phi$ is locked at $\phi=0$ mod $\pi$ to gain the energy [see Eq. (2.19)], and consequently the spin excitations have a gap. On the other hand, if $g_{s}$ $>0$, then $\left|G_{s}(l)\right|$ decreases to zero as $l$ increases, and the $\phi$ field becomes a free field; the spin sector has massless excitations. The approach of $G_{s}$ to zero is very slow $(\sim 1 / l)$, and the $\phi$ field has a strong tendency to be near $\phi=\pi / 2 \bmod \pi$. Although it eventually fails to lock the phase $\phi$, the marginally irrelevant coupling still has an impact on low-energy properties by giving rise to logarithmic corrections to correlation functions. ${ }^{58}$

The charge sector is governed by the two couplings $G_{c}$ and $G_{\rho}$, whose RG flow diagram is of the KosterlitzThouless type. Since $g_{\rho}=(U+6 V) a>0, G_{c}$ is a relevant coupling and always flows to strong-coupling regime, unless $g_{c}=0$. This means that $G_{c}(l)$ has two strong-coupling fixed
TABLE I. Possible ground-state phases and positions of (quasi) locked phase fields determined only from the marginal terms in Eq. (2.19).

\begin{tabular}{lcc}
\hline \hline Phase & $(\theta, \phi)$ & $\left(g_{c}, g_{s}\right)$ \\
\hline SDW & $(0, \pm \pi / 2),(\pi, \pm \pi / 2)$ & $(+,+)$ \\
CDW & $( \pm \pi / 2,0),( \pm \pi / 2, \pi)$ & $(-,-)$ \\
BCDW & $(0,0),(\pi, \pi),(0, \pi),(\pi, 0)$ & $(+,-)$ \\
BSDW & $( \pm \pi / 2, \pm \pi / 2)$ & $(-,+)$ \\
\hline \hline
\end{tabular}

points, $G_{c}(l) \rightarrow \infty$ and $G_{c}(l) \rightarrow-\infty$, depending on its initial value $g_{c}>0$ and $g_{c}<0$. As seen from Eq. (2.19), the relevant $g_{c}$ with positive (negative) sign implies the phase locking of $\theta$ at the position $\theta=0(\pi / 2) \bmod \pi$.

From the above standard arguments, the ground state can be identified by simply looking at the initial value of the coupling constants $g_{c}$ and $g_{s}$. The ground state is classified into four cases as summarized in Table I, and the positions of locked phases $(\theta, \phi)$ for respective cases are shown in Fig. 3.

(i) $g_{s}<0$ and $g_{c}<0$ : The phase fields are locked at $(\theta, \phi)=\left((\pi / 2)+\pi I_{1}, \pi I_{2}\right)$, where $I_{1}$ and $I_{2}$ are integers. In this case, among the order parameters in Eqs. (2.18), only the CDW order parameter has a finite expectation value, and the ground state is found to be the CDW state. Both charge and spin excitations are gapped.

(ii) $g_{s}<0$ and $g_{c}>0$ : The phase fields are locked at $(\theta, \phi)=\left(\pi I_{1}, \pi I_{2}\right)$. The nonvanishing order parameter is then $\mathcal{O}_{\mathrm{BCDW}}$, and the ground state is the BCDW state. Both charge and spin excitations are gapped.

(iii) $g_{s}>0$ and $g_{c}<0$ : The field $\theta$ is locked at $\theta=(\pi / 2)$ $+\pi I_{1}$, and the field $\phi$ tends to be around $\phi=(\pi / 2)+\pi I_{2}$ although it is not locked in the low-energy limit. In this case the dominant correlation is that of the BSDW state. The charge excitations are gapped whereas the spin excitations are gapless.

(iv) $g_{s}>0$ and $g_{c}>0$ : The field $\theta$ is locked at $\theta=\pi I_{1}$, whereas the field $\phi$ tends to be near $\phi=(\pi / 2)+\pi I_{2}$. The dominant correlation is the SDW order. The charge excitations are gapped while the spin excitations are gapless.

Combining the results of Table I and the coupling con-

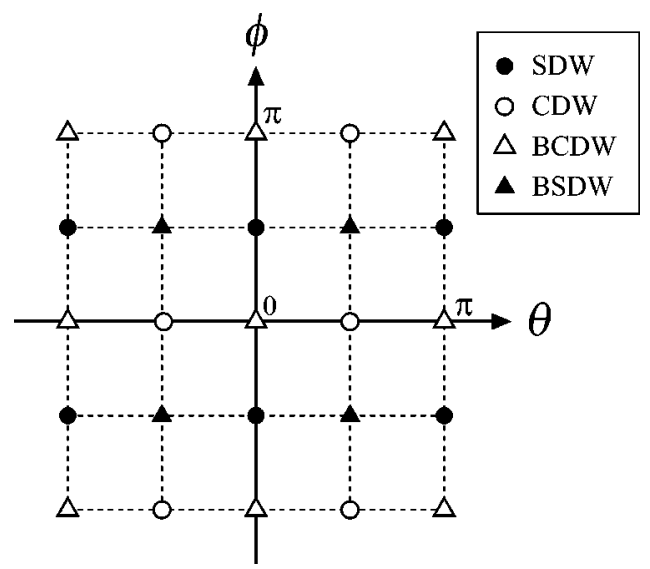

FIG. 3. Positions of locked phase fields $\theta$ and $\phi$ in the SDW, CDW, BCDW, and BSDW states. 
stants Eqs. (2.8a) and (2.8c), we obtain the ground-state phase diagram of the 1D EHM in the weak-coupling limit. For $U$ larger than $2 V$ such that $g_{c}>0$ and $g_{s}>0$, we have the SDW phase, while for $U$ sufficiently smaller than $2 V$ $\left(g_{c}<0\right.$ and $\left.g_{s}<0\right)$ we have the CDW phase. At $U=2 V$, we see from Eqs. (2.8a) and (2.8c) that $g_{s}\left(=g_{1 \perp}\right)<0$ and $g_{c}$ $\left(=g_{3 \perp}\right)>0$ due to the $C_{2}$ term. This implies that a new phase different from the CDW and SDW states appears for $U \approx 2 V$. From Table I, we identify the new phase with the BCDW phase. Within the approximation we employ here, the phase boundary between the BCDW phase and the CDW $(\mathrm{SDW})$ phase is located at $g_{c}=0\left(g_{s}=0\right)$. In this phase diagram, the charge excitations are gapful except on the CDWBCDW transition line, while the spin excitations are gapless in the SDW phase and on the SDW-BCDW transition line. From Eqs. (3.1)-(3.3), we can estimate the charge gap $\Delta_{c}$ and the spin gap $\Delta_{s}$ as

$$
\Delta_{c} \approx t\left(\frac{\left|g_{c}\right|}{t a}\right)^{2 \pi t a / g_{\rho}}, \quad \Delta_{s} \approx t \exp \left(\frac{2 \pi t a}{g_{s}}\right)
$$

for $\left|g_{c}\right| \ll g_{\rho} \ll t a$ and $0<-g_{s} \ll t a$, respectively.

Next we examine effects of the parallel-spin umklapp scattering $g_{c s}$ on the BCDW state. We consider the situation very close to the CDW-BCDW transition by assuming $g_{c}$ $\approx 0$ and $g_{s}<0$, i.e., $U-2 V=-C_{2} V^{2} / \pi t+O\left(V^{3} / t^{2}\right)$. In this case the spin gap is formed first as the energy scale is lowered. For energies below the spin gap, we can replace $\cos 2 \phi$ with its average $\langle\cos 2 \phi\rangle \approx\left(\Delta_{s} / t\right)^{2}$. This means that the coupling constant $g_{c}$ is modified as

$$
g_{c}^{*}=g_{c}+g_{c s}\langle\cos 2 \phi\rangle .
$$

Thus we find that the BCDW state, which is realized for $g_{c}^{*}>0$, becomes less favorable due to the $g_{c s}(<0)$ term. We note, however, that the CDW-BCDW boundary does not move across the $U=2 \mathrm{~V}$ line because $\left|g_{c s}\langle\cos 2 \phi\rangle\right|$ $\approx 2 V a \exp \left[-c(t / V)^{2}\right]$ is much smaller than the $C_{2}$ term in Eq. (2.8c) for $V \ll t$, where $c$ is a positive constant. A similar argument applies to the region near the SDW-BCDW transition. Suppose that $U-2 V=+C_{2} V^{2} / \pi t+O\left(V^{3} / t^{2}\right)$ where $g_{s} \approx 0$ and $g_{c}>0$. In this case, as the energy scale is lowered, the charge gap opens first and the $\theta$ field is pinned at $\theta=0$ mod $\pi$. Below the charge-gap energy scale, the $\phi$ field is subject to the pinning potential $g_{s}^{*} \cos 2 \phi$ with

$$
g_{s}^{*}=g_{s}-g_{c s}\langle\cos 2 \theta\rangle,
$$

where $\langle\cos 2 \theta\rangle \approx\left(\Delta_{c} / t\right)^{2\left(1-G_{\rho}\right)}$. Thus the BCDW phase, which is now realized for $g_{s}^{*}<0$, also becomes less favorable by the $-g_{c s}\langle\cos 2 \theta\rangle(>0)$ term. Again the phase boundary is not moved beyond the $U=2 \mathrm{~V}$ line since $\left|g_{c s}\langle\cos 2 \theta\rangle\right|$ $\approx 2 \mathrm{Va}\left(c^{\prime} V / t\right)^{\pi t / V}$ is much smaller than the $C_{2}$ term in Eq. (2.8a), where $c^{\prime}$ is a constant of order 1 . Therefore we conclude that the BCDW phase is robust against the $g_{c s}$ term in the weak-coupling limit. The analysis in this section establishes the existence of the BCDW phase near $U \approx 2 V$ for 0 $<U, V \ll t$.

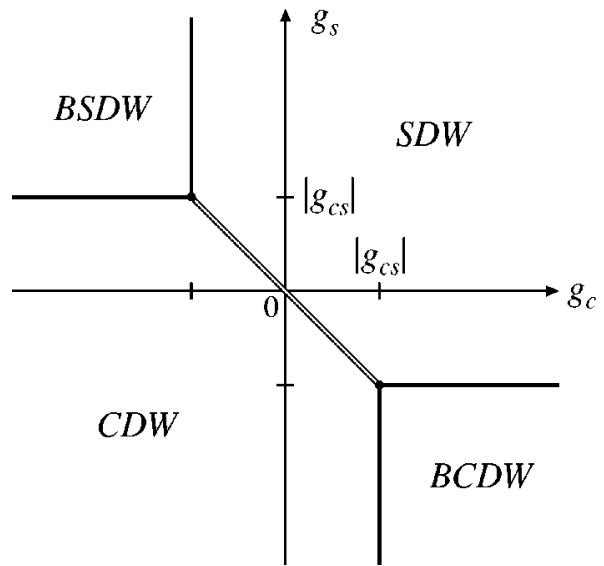

FIG. 4. Phase diagram obtained by minimizing the potential $V(\theta, \phi)$ for $g_{c s}<0$. The double line denotes the first-order transition, while the single line denotes the second-order transition. Bicritical points are at $\left(g_{c}, g_{s}\right)=\left( \pm\left|g_{c s}\right|, \mp\left|g_{c s}\right|\right)$.

\section{B. First-order SDW-CDW transition}

In this section, we discuss how the BCDW phase becomes unstable at strong coupling and how the two continuous transitions change into the first-order SDW-CDW transition.

To our knowledge, Cannon and Fradkin were the first to argue that the $g_{3 \|}$ term (describing the umklapp scattering of parallel-spin electrons), which is conventionally ignored due to its large scaling dimension, can become relevant at large $U$ and $V$ and cause the first-order CDW-SDW transition. ${ }^{22}$ To get an insight into the effect of the $g_{c s}$ term in the relevant case, we perform a semiclassical analysis: we neglect spatial variations of the fields in Eq. (2.19) and focus on the potential,

$$
V(\theta, \phi)=-g_{c} \cos 2 \theta+g_{s} \cos 2 \phi-g_{c s} \cos 2 \theta \cos 2 \phi,
$$

where $g_{c s}=g_{3 \|}<0$. The order parameters of the SDW, CDW, BCDW, and BSDW states take maximum amplitudes when the fields $\theta$ and $\phi$ are pinned at $(\theta, \phi)=\left(\pi I_{1},(\pi / 2)\right.$ $\left.+\pi I_{2}\right), \quad\left((\pi / 2)+\pi I_{1}, \pi I_{2}\right), \quad\left(\pi I_{1}, \pi I_{2}\right), \quad$ and $\quad((\pi / 2)$ $\left.+\pi I_{1},(\pi / 2)+\pi I_{2}\right)$, respectively, where $I_{1}$ and $I_{2}$ are integers. The potential energy in these states is obtained by inserting these pinned fields into Eq. (3.7), e.g., $V_{\mathrm{SDW}}$ $=V\left(\pi I_{1},(\pi / 2)+\pi I_{2}\right)$, yielding

$$
\begin{gathered}
V_{\mathrm{SDW}}=-g_{c}-g_{s}-\left|g_{c s}\right|, \\
V_{\mathrm{CDW}}=+g_{c}+g_{s}-\left|g_{c s}\right|, \\
V_{\mathrm{BCDW}}=-g_{c}+g_{s}+\left|g_{c s}\right|, \\
V_{\mathrm{BSDW}}=+g_{c}-g_{s}+\left|g_{c s}\right| .
\end{gathered}
$$

We find that the $g_{c s}$ term stabilizes the SDW and CDW states while it works against the BCDW and BSDW states. Comparing these energies, we obtain the phase diagram in the $g_{c^{-}}$ $g_{s}$ plane at a fixed $g_{c s}$ (Fig. 4). In the presence of the $g_{c s}$ term, the direct CDW-SDW transition line appears in this phase diagram. 

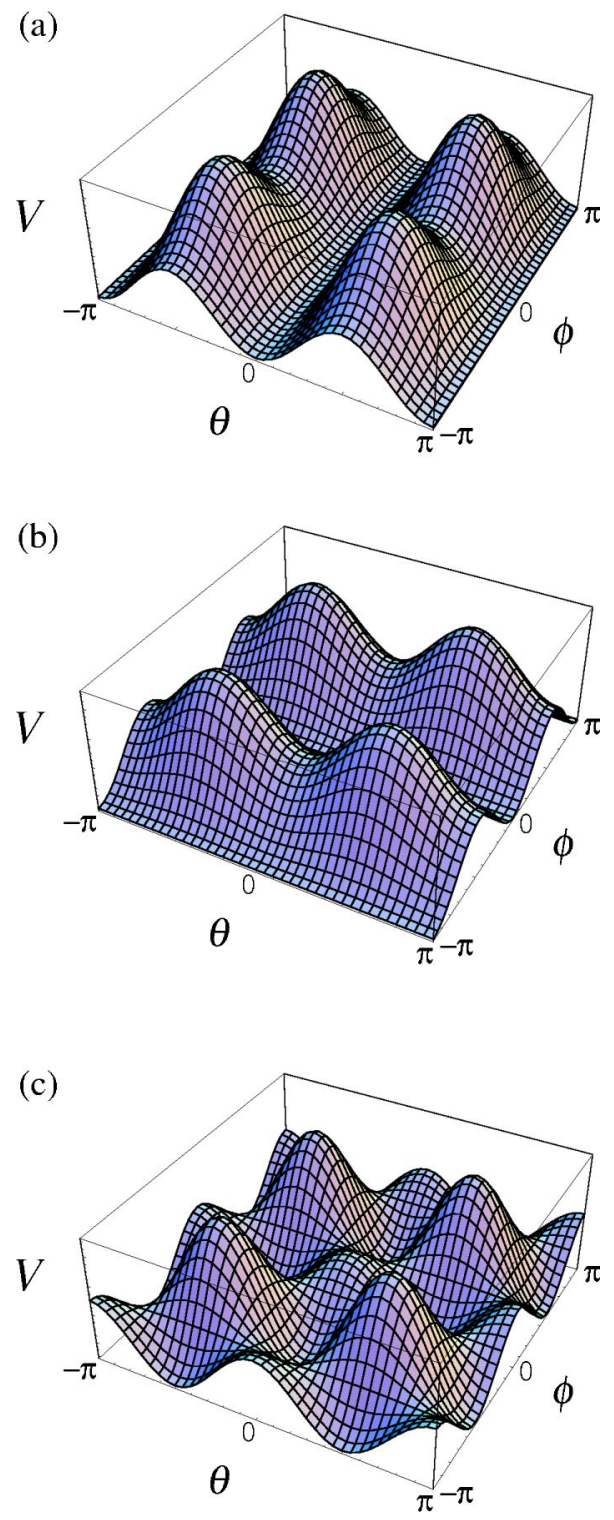

FIG. 5. (Color online) The potential $V(\theta, \phi)$ on the SDWBCDW (a), BCDW-CDW (b), and CDW-SDW (c) transition lines.

We now discuss the nature of the phase transitions. The potential $V(\theta, \phi)$ on various transition lines is shown in Fig. 5. On the boundary between the SDW and BCDW phases, which is located at $g_{s}=-\left|g_{c s}\right|$ and $g_{c}>\left|g_{c s}\right|$, the potential takes the form $V(\theta, \phi)=-g_{c} \cos 2 \theta+g_{s} \cos 2 \phi(1-\cos 2 \theta)$ [Fig. 5(a)], which pins the $\theta$ field at $\theta=\pi I_{1}$ and leaves the $\phi$ field completely free. We thus find that the SDW-BCDW transition is continuous, i.e., the SDW and BCDW phases coexist without potential barrier on the phase boundary. On the boundary between the BCDW and CDW phases, located at $g_{c}=\left|g_{c s}\right|$ and $g_{s}<-\left|g_{c s}\right|$, the potential now takes the form $V(\theta, \phi)=-g_{c} \cos 2 \theta(1-\cos 2 \phi)+g_{s} \cos 2 \phi$ [Fig. 5(b)]. The potential locks the $\phi$ field at $\phi=\pi I_{2}$, where it has no effect on the $\theta$ field. Thus, we find that the CDW-BCDW transition is also continuous. From similar considerations, we find that the SDW-BSDW and BSDW-CDW transitions are continuous as well. In Fig. 4, the phase boundaries of con-

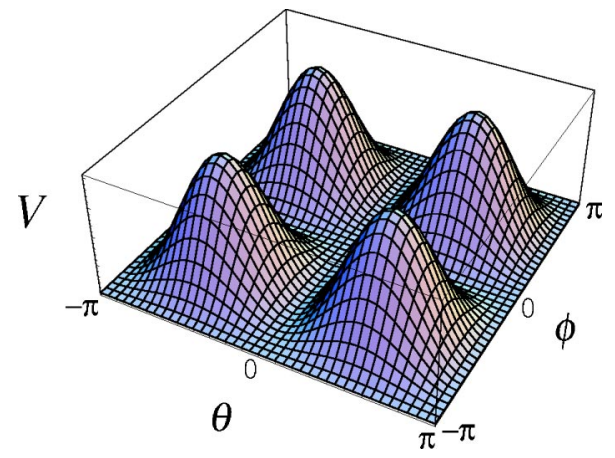

FIG. 6. (Color online) The potential $V(\theta, \phi)$ on the bicritical point $\left(g_{c}, g_{s}\right)=\left(\left|g_{c s}\right|,-\left|g_{c s}\right|\right)$. The potential minima are the lines $\theta=\pi I_{1}$ and $\phi=\pi I_{2}$.

tinuous transitions are shown by the solid lines. On the contrary, the phase boundary shown by the double line in Fig. 4 is of different nature from the others. The potential $V(\theta, \phi)$ on the double line is shown in Fig. 5 (c), where the potential minima are given by the isolated points $(\theta, \phi)$ $=\left(\pi I_{1},(\pi / 2)+\pi I_{2}\right)$ and $\left((\pi / 2)+\pi I_{1}, \pi I_{2}\right)$. These minima correspond to the SDW state and the CDW state, see Fig. 3. The point to note is that there is a finite potential barrier of height $\min \left(\left|g_{c s}\right|, 2\left|g_{c s}\right|-2\left|g_{c}\right|\right)$ between the corresponding minima for the SDW and CDW phases. Hence we conclude that the CDW-SDW transition is first order when $g_{c s}$ is relevant.

From the above arguments, we find that strong umklapp scattering of the parallel-spin electrons destabilizes the BCDW and BSDW states and gives rise to bicritical points $\left(g_{c}, g_{s}\right)= \pm\left(g_{c s},-g_{c s}\right)$ where the two continuous-transition lines merge into the CDW-SDW first-order transition line. Let us take a closer look at these bicritical points. Taking into account the fact that $g_{c}>0$ and $g_{s}<0$ for $U \approx 2 V$ in the original EHM, we will focus on the bicritical point at $\left(g_{c}, g_{s}\right)=\left(\left|g_{c s}\right|,-\left|g_{c s}\right|\right)$. The effective potential at the bicritical point takes the form

$$
V(\theta, \phi)=-g(\cos 2 \theta+\cos 2 \phi-\cos 2 \theta \cos 2 \phi),
$$

which is shown in Fig. 6. This potential has an interesting feature that its potential minima are not isolated points but the crossing lines $\theta=\pi m$ or $\phi=\pi n$ ( $m, n$ : integer). On these lines either $\theta$ or $\phi$ becomes a free field; the theory has more freedom than a single free bosonic field, but less than two free bosonic fields. We thus expect that the theory of the bicritical point should have a central charge larger than 1 but smaller than 2. Detailed analysis of the critical theory is left for a future study. We note that when $g_{c s}=0$ the first-order CDW-SDW transition line collapses into a tetracritical point, $\left(g_{c}, g_{s}\right)=(0,0)$, and the phase boundaries in Fig. 4 reduce to the lines $g_{c}=0$ and $g_{s}=0$ where all the transitions are continuous.

Fabrizio et al. ${ }^{32}$ and Bajnok et al. ${ }^{59}$ discussed effects of higher-frequency terms, such as $\sin 3 \theta$ and $\cos 4 \theta$, which are generated through the renormalization-group transformation. From the semiclassical arguments, it can be seen that these terms can also change a second-order transition to a firstorder transition. ${ }^{59}$ In fact, it was argued that these higher- 


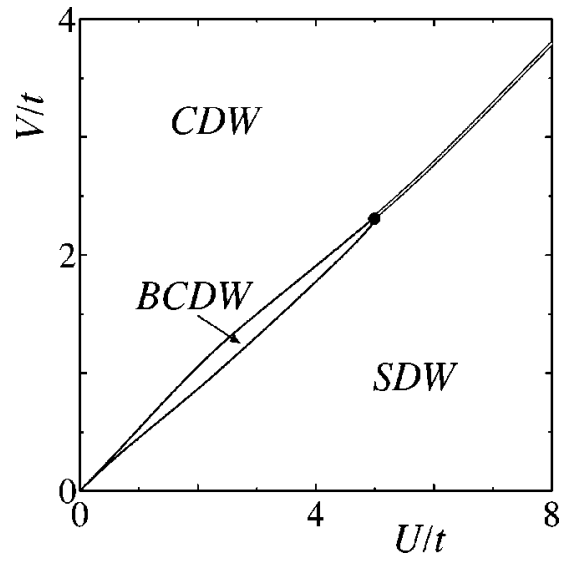

FIG. 7. Phase diagram of the half-filled 1D extended Hubbard model. The double line denotes the first-order transition, while the single lines denote the second-order transitions. The bicritical point is at $\left(U_{c}, V_{c}\right) \approx(5.0 t, 2.3 t)$.

frequency terms make the SDW-CDW transition first order in the strong-coupling regime of the $1 \mathrm{D}$ EHM. ${ }^{32}$ However, we have shown that the SDW-CDW first-order transition can occur simply due to the $g_{c s}$ term which is the leading irrelevant term in this system. Since the higher-frequency terms are even less relevant than the $g_{c s}$ term, we expect that the $g_{c s}$ term should play a dominant role in the first-order transition in the 1D EHM.

\section{Global ground-state phase diagram}

To obtain the global phase diagram of the 1D EHM, we have numerically solved the scaling equations (2.20)-(2.24). We find out which phase is realized by looking at which one of the couplings $G_{c}, G_{s}$, and $G_{c s}$ becomes relevant first, as we have discussed in Secs. III A and III B. First, if $\left|G_{c}\right|$ grows with increasing $l$ and reaches, say, 1 first among the three couplings, then we stop the integration and compute $G_{s}^{*}=G_{s}-G_{c s} \operatorname{sgn}\left(G_{c}\right)$. Since the charge fluctuations are suppressed below this energy scale, we are left with Eq. (3.3), where $G_{s}$ is replaced by $G_{s}^{*}$. We immediately see from Table I that a positive (negative) $G_{s}^{*}$ leads to the SDW (BCDW) state for $G_{c}>0$ and the BSDW (CDW) state for $G_{c}<0$. Second, if $\left|G_{s}\right|$ becomes 1 first, or more precisely, if $G_{s}$ reaches -1 first, then we are left with Eqs. (3.1) and (3.2), where $G_{\rho}$ and $G_{c}$ are replaced by $G_{\rho}^{*}=G_{\rho}-G_{\rho s}$ and $G_{c}^{*}=G_{c}+G_{c s}$, respectively. We see that a positive (negative) $G_{c}^{*}$ leads to the BCDW (CDW) state. Finally, when $\left|G_{c s}\right|$ reaches 1 first, we stop the calculation and compare $G_{c}$ and $G_{s}$. Since both charge and spin fluctuations are already suppressed by the $G_{c s} \cos 2 \theta \cos 2 \phi$ potential, we can deduce the phase from the semiclassical argument. From Fig. 4 we see that we have the SDW state for $G_{s}>-G_{c}$ and the CDW state for $G_{s}<-G_{c}$. Here we note that in the SDW state the pinning potential to the $\phi$ field is marginally irrelevant and thus the spin sector should become gapless.

The phase diagram obtained in this manner is shown in Fig. 7. The single lines denote continuous transitions, and the double line denotes the first-order transition. In the weak- coupling limit, the BCDW phase appears at $U \approx 2 V$ and the successive continuous transitions between the SDW, BCDW, and CDW states occur as $V / U$ increases. When $U$ and $V$ increase along the line $U \approx 2 V$, the BCDW phase first expands and then shrinks up to the bicritical point $\left(U_{c}, V_{c}\right)$ $\approx(5.0 t, 2.3 t)$ where the two continuous-transition lines meet. Beyond this point the BCDW phase disappears and we have the direct first-order transition between the CDW and SDW phases. The phase diagram (Fig. 7) is similar to the ones obtained by using more sophisticated numerical methods. ${ }^{17,25}$ We note that the position of the first-order transition line in Fig. 7 is not reliable quantitatively as we have used the perturbative RG equations. The recent Monte Carlo calculation $^{25}$ gives the most reliable estimate for the position of the bicritical point, $\left(U_{c}, V_{c}\right) \approx((4.7 \pm 0.1) t,(2.51$ $\pm 0.04) t$ ), which agrees with our estimate in Fig. 7 within $10 \%$. The semiquantitative agreement gives us confidence that our approach, semiclassical analysis of the low-energy effective Hamiltonian derived with use of the perturbative $\mathrm{RG}$, is reliable even in the strong-coupling regime near the multicritical point.

\section{EFFECT OF STAGGERED SITE POTENTIAL}

In this section, we examine effects of alternating on-site modulation of the chemical potential, i.e., the staggered site potential, in the half-filled 1D EHM. The Hamiltonian to be considered is given by $H^{\prime}=H+H_{\Delta}$ with $H$ defined in Eq. (2.1) and

$$
H_{\Delta}=\Delta \sum_{j, \sigma}(-1)^{j} n_{j, \sigma}
$$

The model is called the ionic Hubbard model if $V=0$. When $U=V=0$, the system is a trivial band insulator, since the $\Delta$ term induces a gap $2|\Delta|$ at $k= \pm \pi / 2$ in the single-particle spectrum and the lower band is fully filled. For many years effects of the on-site repulsive interaction $U$ on the band insulator have been investigated intensively ${ }^{32-51}$ from both numerical and analytical approaches. Using the standard bosonization method, Fabrizio, Gogolin, and Nersesyan recently argued that the ground state of the ionic Hubbard model exhibits three phases as $U$ increases: the band insulator, the SDI, and the Mott insulator. ${ }^{32}$ The order parameter of the SDI state is nothing but that of the BCDW state, and we can regard the two states as essentially identical. It was also argued that the quantum phase transition from the band insulator to the SDI state belongs to the Ising universality class whereas the other transition from the SDI state to the Mott insulator is of the Kosterlitz-Thouless type. Recent numerical studies, ${ }^{34-41,45}$ however, have reported controversial results on the existence of the SDI phase. Some claimed to find two quantum phase transitions while others found evidences of only one phase transition. With this issue of the SDI phase in mind, in this section we investigate the phase diagram of the 1D extended Hubbard model with the staggered site potential and examine critical properties of the quantum phase transitions. 
We take into account the staggered site potential and the correlation effects on equal footing by treating them as weak perturbations. We use Eq. (2.17) to rewrite $H_{\Delta}$ in the continuum limit as $H_{\Delta}=\int d x \mathcal{H}_{\Delta}$, where w $^{32,33}$

$$
\mathcal{H}_{\Delta}=-\frac{g_{\Delta}}{2(\pi a)^{2}} \sin \theta \cos \phi
$$

with $g_{\Delta}=4 \pi \Delta a$. Note that the CDW order parameter $\mathcal{O}_{\mathrm{CDW}}$ is proportional to $\mathcal{H}_{\Delta}$, and $g_{\Delta}$ can be regarded as an external force coupled to $\mathcal{O}_{\mathrm{CDW}}$. This has the consequence that $\mathcal{O}_{\mathrm{CDW}}$ acquires a nonvanishing expectation value for any finite $U$ and $V$, as long as $g_{\Delta} \neq 0$. In this section we will denote the insulating phase connected to the free-electron band insulator $(U=V=0$ and $\Delta \neq 0)$ by the $\mathrm{BI}$ phase, rather than the CDW phase.

The bosonized form of the Hamiltonian $H^{\prime}$ can be thought of as a generalization of the so-called double sineGordon (DSG) model as $H^{\prime}$ contains sine/cosine terms with different frequencies $(\sin \theta$ and $\cos 2 \theta, \cos \phi$ and $\cos 2 \phi$ ). The DSG theory itself has been investigated intensively ${ }^{32,59,60}$ and shown to have a critical point belonging to the Ising universality class $\left[c=\frac{1}{2}\right.$ conformal field theory $(\mathrm{CFT})]$. To obtain a qualitative understanding of the critical properties in our system, we first perform a semiclassical analysis in a similar way to Sec. III B, before examining the global phase diagram of $H^{\prime}$ with use of the RG method.

\section{A. Semiclassical analysis}

In this section, we perform a semiclassical analysis to the Hamiltonian $\mathcal{H}^{\prime}=\mathcal{H}+\mathcal{H}_{\Delta}$, where $\mathcal{H}$ and $\mathcal{H}_{\Delta}$ are given by Eqs. (2.19) and (4.2), respectively. We neglect spatial variations of the field and focus on the locking potential:

$$
\begin{aligned}
V_{\Delta}(\theta, \phi)= & -g_{c} \cos 2 \theta+g_{s} \cos 2 \phi-g_{c s} \cos 2 \theta \cos 2 \phi \\
& -g_{\Delta} \sin \theta \cos \phi .
\end{aligned}
$$

First, we examine the case $g_{c s}=0$, which corresponds to the situation where the $g_{c s}$ term becomes irrelevant in the RG scheme. The potential to be considered is

$$
\begin{aligned}
V_{\Delta}^{0}(\theta, \phi) & \left.\equiv V_{\Delta}(\theta, \phi)\right|_{g_{c s}=0} \\
& =-g_{c} \cos 2 \theta+g_{s} \cos 2 \phi-g_{\Delta} \sin \theta \cos \phi .
\end{aligned}
$$

Due to its double-frequency structure, possible locations of the phase locking are different from the ones we found in Sec. III B. For example, when $g_{c}>0\left(g_{s}>0\right)$, the two kinds of potentials proportional to $\sin \theta$ and $\cos 2 \theta(\cos \phi$ and $\cos 2 \phi$ ) compete with each other. ${ }^{59,60}$ The locking of the phases $\theta$ and $\phi$ are determined from the saddle-point equations: $\quad \cos \theta\left(4 g_{c} \sin \theta-g_{\Delta} \cos \phi\right)=0 \quad$ and $\quad \sin \phi\left(-4 g_{s} \cos \phi\right.$ $\left.+g_{\Delta} \sin \theta\right)=0$. In order to simplify the notations, let us introduce

$$
\alpha_{\theta}^{0} \equiv\left|\cos ^{-1}\left(\frac{g_{\Delta}}{4 g_{c}}\right)\right|, \quad \alpha_{\phi}^{0} \equiv\left|\cos ^{-1}\left(\frac{g_{\Delta}}{4 g_{s}}\right)\right|,
$$

TABLE II. Possible ordered ground states and the position of (quasi)locked phase fields determined from Eq. (4.4).

\begin{tabular}{lc}
\hline \hline \multicolumn{1}{c}{ Phase } & $(\theta, \phi)$ \\
\hline SDW & $(0, \pm \pi / 2),(\pi, \pm \pi / 2)$ \\
BI (for $\left.g_{\Delta}>0\right)$ & $(+\pi / 2,0),(-\pi / 2, \pi)$ \\
BI (for $\left.g_{\Delta}<0\right)$ & $(+\pi / 2, \pi),(-\pi / 2,0)$ \\
BCDW & $\left(+(\pi / 2) \pm \alpha_{\theta}^{0}, 0\right),\left(-(\pi / 2) \pm \alpha_{\theta}^{0}, \pi\right)$ \\
BSDW & $\left(+\pi / 2,0 \pm \alpha_{\phi}^{0}\right),\left(-\pi / 2, \pm\left(\pi-\alpha_{\phi}^{0}\right)\right)$ \\
\hline \hline
\end{tabular}

where $\left|g_{\Delta} / g_{c}\right| \leqslant 4,\left|g_{\Delta} / g_{s}\right| \leqslant 4$, and $0 \leqslant \alpha_{\theta}^{0}, \alpha_{\phi}^{0} \leqslant \pi$ are assumed. The solutions of the saddle-point equations yield the following four states with distinct configurations of the locked phase fields $\theta$ and $\phi$ (modulo $2 \pi$ ): (i) the SDW state with $\theta$ and $\phi$ locked at $(\theta, \phi)=(0, \pm \pi / 2)$ or $(\pi, \pm \pi / 2)$; (ii) the BI state with $(\theta, \phi)=(+\pi / 2,0),(-\pi / 2, \pi)$ if $g_{\Delta}>0$ and with $(\theta, \phi)=(+\pi / 2, \pi), \quad(-\pi / 2,0)$ if $g_{\Delta}<0$; (iii) the "BCDW" state where the BCDW order and the CDW order coexist and which is realized when $(\theta, \phi)=\left(\pi / 2 \pm \alpha_{\theta}^{0}, 0\right)$ or $\left(-\pi / 2 \pm \alpha_{\theta}^{0}, \pi\right)$; (iv) the "BSDW" state where the BSDW and the CDW order coexist and which is realized when $(\theta, \phi)=\left(\pi / 2,0 \pm \alpha_{\phi}^{0}\right)$ or $\left(-\pi / 2, \pm\left(\pi-\alpha_{\phi}^{0}\right)\right)$. Table II and Fig. 8 summarize the possible ordered ground states and corresponding positions of locked phase fields. The potential energies in these states are given by

$$
\begin{gathered}
V_{\mathrm{SDW}}^{0}=-g_{c}-g_{s}, \\
V_{\mathrm{BI}}^{0}=+g_{c}+g_{s}-\left|g_{\Delta}\right|, \\
V_{\mathrm{BCDW}}^{0}=-g_{c}+g_{s}-\frac{g_{\Delta}^{2}}{8 g_{c}}, \\
V_{\mathrm{BSDW}}^{0}=+g_{c}-g_{s}-\frac{g_{\Delta}^{2}}{8 g_{s}} .
\end{gathered}
$$

In deriving Eqs. (4.6c) and (4.6d), we have assumed $\left|g_{\Delta} / g_{c}\right| \leqslant 4$ and $\left|g_{\Delta} / g_{s}\right| \leqslant 4$, respectively. The CDW state is

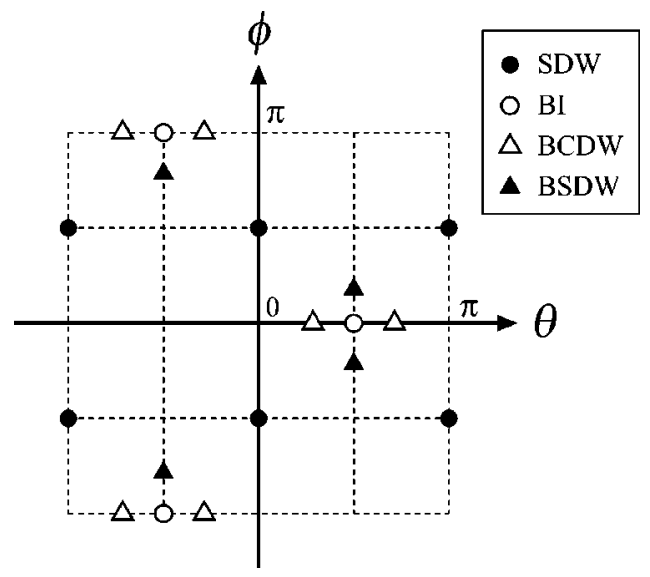

FIG. 8. Positions of locked phase fields $\theta$ and $\phi$ in the four states when $g_{\Delta}>0$. 


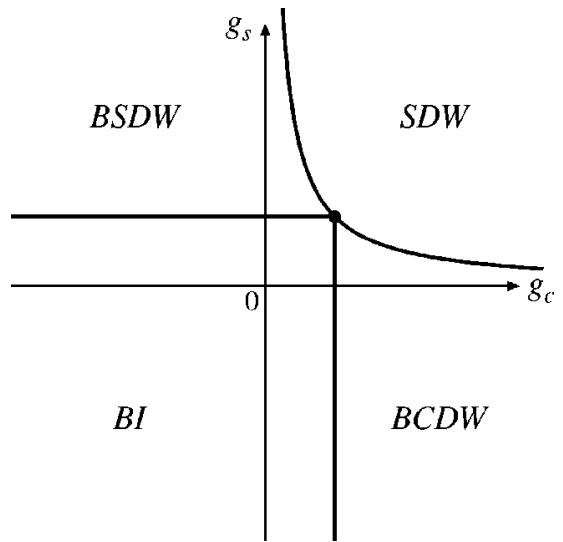

FIG. 9. Phase diagram obtained by minimizing the potential energy $V_{\Delta}^{0}(\theta, \phi)$ [Eq. (4.4)]. The phase boundaries between the SDW state and the BCDW state, and between the SDW state and the BSDW state are given by the curve $g_{s}=g_{\Delta}^{2} /\left(16 g_{c}\right)$ with $g_{c}$ $>0$. The phase boundaries between the BI state and the BCDW state, and between the BI state and the BSDW state are given by the lines $g_{c}=\frac{1}{4}\left|g_{\Delta}\right|$ with $g_{s}<\frac{1}{4}\left|g_{\Delta}\right|$ and $g_{s}=\frac{1}{4}\left|g_{\Delta}\right|$ with $g_{c}<\frac{1}{4}\left|g_{\Delta}\right|$, respectively. All the phase transitions in this figure are continuous. The tetracritical point is located at $\left(g_{c}, g_{s}\right)=\left(\frac{1}{4}\left|g_{\Delta}\right|, \frac{1}{4}\left|g_{\Delta}\right|\right)$.

stabilized strongly by the $g_{\Delta}$ term whereas the BCDW state and the BSDW state are also stabilized by the second-order contribution of $g_{\Delta}$. By comparing these energies, we arrive at the phase diagram shown in Fig. 9. As we go across the boundary $\left(g_{c}=\frac{1}{4} g_{\Delta}\right)$ from the BI state to the BCDW state, we find that each potential minimum splits into two minima, e.g., $(\theta, \phi)=(\pi / 2,0) \rightarrow\left((\pi / 2) \pm \alpha_{\theta}^{0}, 0\right)$, and that the potential for the $\theta$ phase field takes a double-well structure in the BCDW state. Similarly, as we go from the BI state to the BSDW state, each potential minimum splits into two minima, e.g., $(\theta, \phi)=(\pi / 2,0) \rightarrow\left(\pi / 2, \pm \alpha_{\phi}^{0}\right)$, and now the potential for the $\phi$ phase field has a double-well structure in the BSDW state. As long as $g_{c s}=0$, any quantum phase transition is continuous since a potential barrier between two potential minima corresponding to two different states vanishes at the transition. The phase diagram (Fig. 9) indicates that a direct transition from the SDW state to the BI state takes place only when the parameters $g_{c}$ and $g_{s}$ are on the multicritical point $\left(g_{c}, g_{s}\right)=\left(\frac{1}{4}\left|g_{\Delta}\right|, \frac{1}{4}\left|g_{\Delta}\right|\right)$, where the potential takes the form $V_{\Delta}^{0}(\theta, \phi)=\frac{1}{2}\left|g_{\Delta}\right|\{-1+[\sin \theta$ $\left.\left.-\operatorname{sgn}\left(g_{\Delta}\right) \cos \phi\right]^{2}\right\}$ and is minimized at $\phi= \pm[(\pi / 2)-\theta]$ and $\phi= \pm\left(\frac{3}{2} \pi+\theta\right)$ if $g_{\Delta}>0$, or at $\phi= \pm[(\pi / 2)+\theta]$ and $\phi= \pm\left(\frac{3}{2} \pi-\theta\right)$ if $g_{\Delta}<0$.

Let us take a closer look at low-energy excitations in the BI state and the BCDW state. The massive sine-Gordon model has topological excitations, solitons, and antisolitons. They are characterized by the topological charges $Q$ and $S_{z}$ for the charge and the spin sectors,

$$
Q=\frac{1}{\pi} \int d x \partial_{x} \theta, \quad S_{z}=\frac{1}{2 \pi} \int d x \partial_{x} \phi .
$$

In the noninteracting case $(U=V=0)$ with a finite $\Delta$, the lowest-energy excitation is a soliton of $\theta$ and $\phi$ connecting two neighboring minima of the $-g_{\Delta} \sin \theta \cos \phi$, e.g., $\left.(\theta, \phi)\right|_{x \rightarrow-\infty}=(-\pi / 2, \pi)$ and $\left.(\theta, \phi)\right|_{x \rightarrow \infty}=(\pi / 2,0)$. Such an excitation carries the charge $Q= \pm 1$ and the spin $S_{z}= \pm \frac{1}{2}$, which is nothing but a single-electron excitation in the band insulator. It has been pointed out ${ }^{32,46,47}$ that in the SDI phase (i.e., in the BCDW phase), the topological charge $Q$ of the lowest-energy excitation becomes fractional, $Q= \pm 2 \alpha_{\theta}^{0} / \pi$, reflecting the local double-well structure of the potential near the potential minima, e.g., at $(\theta, \phi)=\left(\pi / 2 \pm \alpha_{\theta}^{0}, 0\right)$. This is a unique feature of the BCDW phase and is contrasted from the integer charge $Q= \pm 1$ of the lowest-energy excitation in the pure BCDW phase where the phase fields are locked at $(\theta, \phi)=(0,0)$. Accordingly, the phase transition between the BCDW state and the BI state belongs to a different universality class from the one between the pure BCDW state and the CDW state discussed in Sec. III B. In the former case, a small potential barrier in a double-well potential in the BCDW state vanishes at the critical point and the effective theory for the low-energy excitations is the " $\varphi$ ", theory known to describe the Ising phase transition, rather than the Gaussian theory that governs the transition between the BCDW and CDW phases.

One might expect that a similar semiclassical analysis can be applied to the spin field $\phi$. Within the semiclassical approach the topological charge $S_{z}$ in the BSDW phase of Fig. 9 takes a fractional value, $\pm \alpha_{\phi}^{0} /(2 \pi)$. However, since the Hamiltonian has the global SU(2) spin-rotation symmetry, the SDW state and the BSDW state cannot have a true longrange order. This implies that the phase field $\phi$ cannot be localized except in spin-gap phases where $\phi$ is locked at $\langle\phi\rangle=0 \bmod \pi$. The global $\mathrm{SU}(2)$ symmetry thus prohibits the Ising criticality in the spin sector. In fact, the BSDW phase in Fig. 9 turns out to be just the BI phase.

Let us now consider the situation in which $g_{c s} \neq 0$. In this case, the phase fields $\theta$ and $\phi$ are locked in a similar way to the case $g_{c s}=0$, but $\alpha_{\theta}^{0}$ and $\alpha_{\phi}^{0}$ are modified into $\alpha_{\theta}^{0} \rightarrow \alpha_{\theta}$ and $\alpha_{\phi}^{0} \rightarrow \alpha_{\phi}$, where

$$
\begin{aligned}
& \alpha_{\theta} \equiv\left|\cos ^{-1}\left[\frac{g_{\Delta}}{4\left(g_{c}-\left|g_{c s}\right|\right)}\right]\right|, \\
& \alpha_{\phi} \equiv\left|\cos ^{-1}\left[\frac{g_{\Delta}}{4\left(g_{s}-\left|g_{c s}\right|\right)}\right]\right| .
\end{aligned}
$$

Here we have assumed $\left|g_{\Delta} /\left(g_{c}-\left|g_{c s}\right|\right)\right| \leqslant 4$ and $\mid g_{\Delta} /\left(g_{s}\right.$ $\left.-\left|g_{c s}\right|\right) \mid \leqslant 4$. The potential energies in the four states become

$$
\begin{gathered}
V_{\mathrm{SDW}}=-g_{c}-g_{s}-\left|g_{c s}\right|, \\
V_{\mathrm{BI}}=+g_{c}+g_{s}-\left|g_{c s}\right|-\left|g_{\Delta}\right|, \\
V_{\mathrm{BCDW}}=-g_{c}+g_{s}+\left|g_{c s}\right|-\frac{g_{\Delta}^{2}}{8\left(g_{c}-\left|g_{c s}\right|\right)}, \\
V_{\mathrm{BSDW}}=+g_{c}-g_{s}+\left|g_{c s}\right|-\frac{g_{\Delta}^{2}}{8\left(g_{s}-\left|g_{c s}\right|\right)} .
\end{gathered}
$$




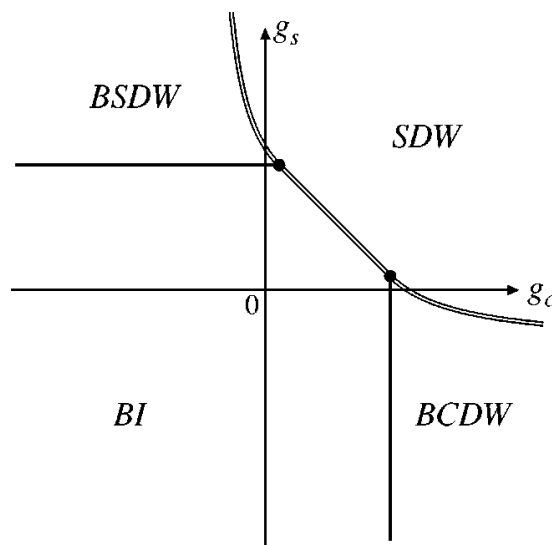

FIG. 10. Phase diagram obtained by minimizing the potential energy $V_{\Delta}(\theta, \phi)$ [Eq. (4.3)] for $g_{c s}<0$. The phase boundaries are given by $g_{s}=-g_{c}+\frac{1}{2}\left|g_{\Delta}\right|$ between the SDW and the BI states, $g_{c}=\left|g_{c s}\right|+\frac{1}{4}\left|g_{\Delta}\right|$ between the BI and the BCDW states, $g_{s}=\left|g_{c s}\right|$ $+\frac{1}{4}\left|g_{\Delta}\right|$ between the BI and the BSDW states, $g_{s}=-\left|g_{c s}\right|$ $+g_{\Delta}^{2} /\left[16\left(g_{c}-\left|g_{c s}\right|\right)\right]$ between the SDW and the BCDW states, and $g_{c}=-\left|g_{c s}\right|+g_{\Delta}^{2} /\left[16\left(g_{s}-\left|g_{c s}\right|\right)\right]$ between the SDW and the BSDW states. Multicritical points are located at $\left(g_{c}, g_{s}\right)=\left(+\mid g_{c s}\right.$ $\left.\left|+\frac{1}{4}\right| g_{\Delta}|,-| g_{c s}\left|+\frac{1}{4}\right| g_{\Delta} \mid\right)$ and $\left(-\left|g_{c s}\right|+\frac{1}{4}\left|g_{\Delta}\right|,+\left|g_{c s}\right|+\frac{1}{4}\left|g_{\Delta}\right|\right)$. The single lines denote second-order transitions, while the double lines denote first-order transitions.

By comparing these energies we obtain the phase diagram (Fig. 10). In the limit $g_{\Delta} \rightarrow 0$ this phase diagram reduces to Fig. 4. One can easily find that the $g_{c s}$ term favors the SDW state and the BI state over the BCDW state and the BSDW state. The direct SDW-BI transition line acquires a finite length in the phase diagram, like in Fig. 4. The analysis of critical properties of each quantum phase transition is more complicated than that in Sec. III due to the presence of two kinds of charge-spin coupled terms, the $g_{\Delta}$ and $g_{c s}$ terms. Along the phase boundary between the SDW state and the BI state, the potential energy is minimized at discrete points, $(\theta, \phi)=(-\pi / 2, \pi), \quad(0, \pm \pi / 2), \quad(\pi / 2,0), \quad(\pi, \pm \pi / 2)$ for $g_{\Delta}>0, \quad$ or at $(\theta, \phi)=(-\pi / 2,0), \quad(0, \pm \pi / 2), \quad(\pi / 2, \pi)$, $(\pi, \pm \pi / 2)$ for $g_{\Delta}<0$. These points correspond either to the SDW state or to the BI state (see Table II). Since any path connecting these potential minima has to go over a potential barrier, the direct SDW-BI transition is first order. In addition, both the transition between the SDW state and the BCDW state and that between the SDW state and the BSDW state become first order when $g_{c s} \neq 0$. On the phase boundary between the SDW state and the BCDW state, the potential has isolated minima at $(\theta, \phi)=(0, \pm \pi / 2),(\pi, \pm \pi / 2)$, $\left(-\pi / 2 \pm \alpha_{\theta}, \pi\right)$, and $\left(+\pi / 2 \pm \alpha_{\theta}, 0\right)$. The pinning of the phase fields at these minima corresponds either to the SDW state or to the BCDW state (see Fig. 8). On the multicritical points at $\left(g_{c}, g_{s}\right)=\left(+\left|g_{c s}\right|+\frac{1}{4}\left|g_{\Delta}\right|,-\left|g_{c s}\right|+\frac{1}{4}\left|g_{\Delta}\right|\right)$ and $\left(-\left|g_{c s}\right|+\frac{1}{4}\left|g_{\Delta}\right|,+\left|g_{c s}\right|+\frac{1}{4}\left|g_{\Delta}\right|\right)$, the potential takes the form

$$
\begin{aligned}
V_{\Delta}^{c 1}(\theta, \phi)= & -\left|g_{c s}\right|(\cos 2 \theta+\cos 2 \phi-\cos 2 \theta \cos 2 \phi) \\
& +\frac{1}{2}\left|g_{\Delta}\right|\left\{-1+\left[\sin \theta-\operatorname{sgn}\left(g_{\Delta}\right) \cos \phi\right]^{2}\right\},
\end{aligned}
$$

$$
\begin{aligned}
V_{\Delta}^{c 2}(\theta, \phi)= & +\left|g_{c s}\right|(\cos 2 \theta+\cos 2 \phi+\cos 2 \theta \cos 2 \phi) \\
& +\frac{1}{2}\left|g_{\Delta}\right|\left\{-1+\left[\sin \theta-\operatorname{sgn}\left(g_{\Delta}\right) \cos \phi\right]^{2}\right\},
\end{aligned}
$$

respectively. The potential minima of $V_{\Delta}^{c 1}(\theta, \phi)$ and $V_{\Delta}^{c 2}(\theta, \phi)$ are located at $(\theta, \phi)=(-\pi / 2, \pi),(0, \pm \pi / 2)$, $(\pi / 2,0), \quad$ and $(\pi, \pm \pi / 2)$ for $g_{\Delta}>0$ and at $(\theta, \phi)$ $=(-\pi / 2,0),(0, \pm \pi / 2),(\pi / 2, \pi)$, and $(\pi, \pm \pi / 2)$ for $g_{\Delta}$ $<0$.

Finally, we note that even in the SDW state (the Mott insulator) the CDW order parameter has a nonvanishing expectation value. This is because the alternating site potential $\mathcal{H}_{\Delta}$ has the same form as the CDW order parameter $\mathcal{O}_{\mathrm{CDW}}$ $\propto \sin \theta \cos \phi$. Even though the semiclassical analysis indicates that the phase fields are pinned, say, at $(\theta, \phi)=(0$, $\pm \pi / 2$ ), quantum fluctuations of the fields around the pinning position lead to a nonvanishing $\left\langle\mathcal{O}_{\mathrm{CDW}}\right\rangle$. This can be easily seen in the limit of small $\Delta$, where

$$
\begin{aligned}
\left\langle O_{\mathrm{CDW}}\right\rangle & \propto \operatorname{Tr}\left[\exp \left[-\int d x\left(\mathcal{H}+\mathcal{H}_{\Delta}\right)\right] \sin \theta \cos \phi\right] \\
& \propto g_{\Delta} \operatorname{Tr}\left[\exp \left(-\int d x \mathcal{H}\right) \sin ^{2} \theta \cos ^{2} \phi\right] \neq 0 .
\end{aligned}
$$

\section{B. Renormalization-group analysis}

We perform $\mathrm{RG}$ analysis to take into account quantum fluctuations that are ignored in the semiclassical analysis. As in Sec. III, we obtain the RG equations using the OPE method (see Appendix B):

$$
\begin{gathered}
\frac{d}{d l} G_{\Delta}=+G_{\Delta}+\frac{1}{2} G_{\Delta} G_{\rho}-G_{\Delta} G_{c}-\frac{3}{2} G_{\Delta} G_{s} \\
-\frac{3}{4} G_{\Delta} G_{c s}-\frac{3}{8} G_{\Delta} G_{\rho s} \\
\frac{d}{d l} G_{\rho}=+\frac{1}{4} G_{\Delta}^{2}+2 G_{c}^{2}+G_{c s}^{2}+G_{s} G_{\rho s} \\
\frac{d}{d l} G_{c}=-\frac{1}{4} G_{\Delta}^{2}+2 G_{\rho} G_{c}-G_{s} G_{c s}-G_{c s} G_{\rho s} \\
\frac{d}{d l} G_{s}=-\frac{1}{4} G_{\Delta}^{2}-2 G_{s}^{2}-G_{c} G_{c s}-G_{c s}^{2} \\
\frac{d}{d l} G_{c s}=-\frac{1}{4} G_{\Delta}^{2}-2 G_{c s}+2 G_{\rho} G_{c s}-4 G_{s} G_{c s}-2 G_{c} G_{s} \\
-2 G_{c} G_{\rho s}-4 G_{c s} G_{\rho s},
\end{gathered}
$$




$$
\begin{aligned}
\frac{d}{d l} G_{\rho s}= & -\frac{1}{4} G_{\Delta}^{2}-2 G_{\rho s}+2 G_{\rho} G_{s}-4 G_{c} G_{c s} \\
& -4 G_{c s}^{2}-4 G_{s} G_{\rho s} .
\end{aligned}
$$

The initial value of $G_{\Delta}(l)$ is given by $G_{\Delta}(0)=\Delta / t$, while those of the other coupling constants are given by $G_{\nu}(0)$ $=g_{\nu} /(4 \pi t a)$. Since the RG equations are invariant under the sign change of $G_{\Delta}\left(G_{\Delta} \rightarrow-G_{\Delta}\right)$, we can assume $G_{\Delta}(0) \geqslant 0$ without losing generality in the following arguments.

We determine the ground-state phase diagram in a similar way as in Sec. III. That is, we integrate the scaling equations (4.12)-(4.17) numerically and find which one of the couplings $\left[G_{\Delta}(l), G_{c}(l), G_{s}(l)\right.$, and $\left.G_{c s}(l)\right]$ becomes most relevant. By doing so, we have encountered the following four cases.

(i) The case where $G_{c}(l)$ grows fastest and becomes 1 at $l=l_{\rho+}$. Below this energy scale (i.e., $l \geqslant l_{\rho+}$ ), the charge fluctuations are suppressed and the phase field $\theta$ is locked at $\theta=0$ or $\pi$. For the discussion of the ground-state properties we may first neglect the $g_{\Delta}$ term since $\langle\sin \theta\rangle \cos \phi=0$. The Hamiltonian density $\mathcal{H}^{\prime}$ then reduces to

$$
\begin{aligned}
\mathcal{H}_{\sigma+}^{\mathrm{eff}}= & \frac{v_{F}}{2 \pi} \sum_{p}\left(\partial_{x} \phi_{p}\right)^{2}-\frac{v_{F}}{\pi} G_{s}^{*}\left(\partial_{x} \phi_{+}\right)\left(\partial_{x} \phi_{-}\right) \\
& +\frac{v_{F}}{\pi a^{2}} G_{s}^{*} \cos 2 \phi,
\end{aligned}
$$

where $G_{s}^{*}=G_{s}\left(l_{\rho+}\right)-G_{c s}\left(l_{\rho+}\right)$. We immediately see that, if $G_{s}^{*}>0$, the spin excitations are gapless and the ground state is the SDW state. On the other hand, if $G_{s}^{*}<0$, then the operators proportional to $G_{s}^{*}$ are relevant $\left[G_{s}^{*}(l) \rightarrow-\infty\right.$ under scaling] and the phase fields are locked as $(\theta, \phi)$ $=(0,0),(0, \pi),(\pi, 0),(\pi, \pi)$, which corresponds to the BCDW state with $\alpha_{\theta} \rightarrow \pi / 2$ (i.e., $g_{\Delta} \rightarrow 0$ ), see Table II. This would become the BCDW state with $\alpha_{\theta}<\pi / 2$ in a more realistic treatment where the $g_{\Delta}$ term is not simply ignored.

(ii) The case where $\left|G_{c}(l)\right|$ grows most rapidly and $G_{c}(l) \rightarrow-1$ at $l=l_{\rho^{-}}$. The phase field $\theta$ is then locked at $\theta= \pm \pi / 2$ for $l>l_{\rho_{-}}$. Below this energy scale one can replace the $\sin \theta$ potential by its averaged value, i.e., $\sin \theta$ $\rightarrow\langle\sin \theta\rangle= \pm 1$. The effective Hamiltonian at $l=l_{\rho^{-}}$is given by

$$
\begin{aligned}
\mathcal{H}_{\sigma-}^{\mathrm{eff}}= & \frac{v_{F}}{2 \pi} \sum_{p}\left(\partial_{x} \phi_{p}\right)^{2}-\frac{v_{F}}{\pi} G_{s}^{*}\left(\partial_{x} \phi_{+}\right)\left(\partial_{x} \phi_{-}\right) \\
& \mp \frac{v_{F}}{\pi a^{2}} G_{\Delta}^{*} \cos \phi+\frac{v_{F}}{\pi a^{2}} G_{s}^{*} \cos 2 \phi,
\end{aligned}
$$

where $G_{\Delta}^{*}=G_{\Delta}\left(l_{\rho^{-}}\right)$and $G_{s}^{*}=G_{s}\left(l_{\rho^{-}}\right)+G_{c s}\left(l_{\rho^{-}}\right)$, and the sign $-/+$ of the $G_{\Delta}$ term corresponds to the position of the phase locking $\theta=+(\pi / 2) /-(\pi / 2)$. When $G_{s}^{*}>0$, the two $G_{s}^{*}$ terms are marginally irrelevant, and the only relevant operator is $\mp \cos \phi$. Then the phase field $\phi$ is locked at $\phi$ $=0$ or $\pi$, depending on the position of the charge phase locking $\theta=+(\pi / 2)$ or $-(\pi / 2)$. On the other hand, when $G_{s}^{*}<0$, both $G_{\Delta}^{*}$ and $G_{s}^{*}$ terms become relevant. However, these terms do not compete with each other. The only effect of the $G_{\Delta}^{*}$ term is to lift the degeneracy between the neighboring minima of $-\cos 2 \phi$, and hence the position of the phase locking is the same as in the case $G_{s}^{*}>0$. Therefore, regardless of the sign of $G_{s}^{*}$, the resultant phase is found to be the BI state with the phase locking at $(\theta, \phi)=(\pi / 2,0)$ or $(-\pi / 2, \pi)$.

(iii) The case where either $\left|G_{c s}(l)\right|$ or $\left|G_{\Delta}(l)\right|$ is most relevant. Then both charge and spin fluctuations are suppressed, and the classical treatment is sufficient at lower energy scale. In this case, we find to which phase the ground state belongs by substituting the parameters $G_{c}(l)$ and $G_{s}(l)$ into $g_{c}$ and $g_{s}$ in Fig. 10.

(iv) The case where $G_{s}(l)$ is most relevant and becomes -1 at $l=l_{\sigma}$. Below this energy scale the spin fluctuations are suppressed and the phase field $\phi$ is locked as $\phi \rightarrow 0$ or $\pi$ for $l>l_{\sigma}$. The effective Hamiltonian of the remaining charge sector is

$$
\begin{aligned}
\mathcal{H}_{\rho}^{\mathrm{eff}}= & \frac{v_{F}}{2 \pi} \sum_{p}\left(\partial_{x} \theta_{p}\right)^{2}+\frac{v_{F}}{\pi} G_{\rho}^{*}\left(\partial_{x} \theta_{+}\right)\left(\partial_{x} \theta_{-}\right) \\
& \mp \frac{v_{F}}{\pi a^{2}} G_{\Delta}^{*} \sin \theta-\frac{v_{F}}{\pi a^{2}} G_{c}^{*} \cos 2 \theta,
\end{aligned}
$$

where $G_{\rho}^{*}=G_{\rho}\left(l_{\sigma}\right)-G_{\rho s}\left(l_{\sigma}\right), \quad G_{\Delta}^{*}=G_{\Delta}\left(l_{\sigma}\right), \quad$ and $G_{c}^{*}$ $=G_{c}\left(l_{\sigma}\right)+G_{c s}\left(l_{\sigma}\right)$. The sign $-/+$ of the $G_{\Delta}^{*}$ term corresponds to the position of the phase locking $\phi=0 / \pi$. In this Hamiltonian, both of the nonlinear terms, $\sin \theta$ and $\cos 2 \theta$, are relevant operators. If $G_{c}^{*}<0$, then the situation is the same as the case (ii): the $G_{\Delta}^{*}$ and $G_{c}^{*}$ terms do not compete with each other and the possible phase locking pattern is $\theta$ $=+\pi / 2(-\pi / 2)$ for $\phi=0(\pi)$, where the ground state is the BI state. If $G_{c}^{*}>0$, these two terms compete with each other, since the $-(+) \sin \theta$ potential tends to lock the phase field $\theta$ at $\theta=+\pi / 2(-\pi / 2)$, while the $\cos 2 \theta$ potential tends to lock it at $\theta=0$ or $\pi$. In this case, possible ground states are the BI state and the BCDW state, and the quantum phase transition between them is of the Ising-transition type with the central charge $c=1 / 2$, as discussed in the preceding section. However, it is hard to estimate quantitatively the critical value of the coupling constants at the quantum phase transition. One way to estimate it is to find a critical point separating the basins of attraction to the two strong-coupling fixed points, $\left(G_{\Delta}^{*}, G_{c}^{*}\right) \rightarrow(+\infty,-\infty)$ and $(0,+\infty)$, in the perturbative RG analysis. ${ }^{33,61}$ However, with this method where the cosine and sine terms are treated perturbatively, we cannot see the correct picture of the DSG theory with the double-well potential structure which leads to the Ising transition. Instead, here we estimate the critical value for the Ising transition from the semiclassical arguments: The critical value is determined from the condition $G_{c}^{*} / G_{\Delta}^{*}=1 / 4$.

We have used the above scheme to obtain the phase diagram shown in Fig. 11, for which $\Delta / t=0.1$. The phase diagram at large $U$ and $V$ is similar to Fig. 7, whereas a qualitative charge in the phase diagram is found in the region 


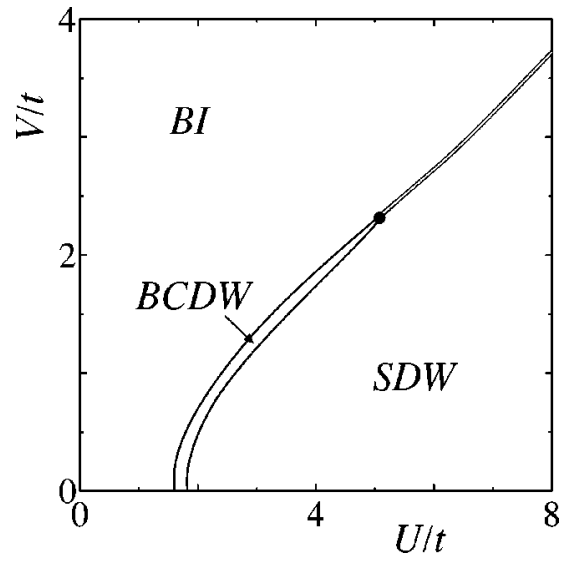

FIG. 11. Phase diagram of the half-filled extended Hubbard model at $\Delta / t=0.1$. The double line denotes the first-order transition, while the single lines denote the second-order transitions.

$U, V \lesssim t$. In agreement with Fabrizio, Gogolin, and Nersesyan, ${ }^{32}$ we obtain two critical points $\left(U_{c 1}<U_{c 2}\right)$ separating three phases on the $U$ axis: the $\mathrm{BI}$ state, the BCDW state ( = the SDI state ${ }^{32}$ ), and the SDW state. From comparison of Figs. 7 and 11, we see that the BCDW state in Fig. 7 has evolved continuously into the BCDW state when the alternating site potential $\Delta$ is switched on. The phase diagram in the $\Delta-V$ plane is shown in Fig. 12, where $U / t=1$. Both $\Delta$ and $V$ promote the BI state, while the SDW ground state is obtained for small $\Delta(\ll U)$ and $V(\ll U)$. We find that the region of the BCDW state obtained in the EHM at $\Delta$ $=0$ is connected to the region of the BCDW state in the Hubbard model with alternating site potential at $V=0$.

Let us discuss in more detail the critical regime in the limit of small $U, V$, and $\Delta$. In this region we can safely neglect the irrelevant terms and set $G_{c s}(l)=G_{\rho s}(l)=0$ in the RG equations (4.12)-(4.17). First we consider the case $V$ $=0$. Integrating out the RG equations (4.12)-(4.15) analytically and following the criterion discussed above, we obtain asymptotic expansion of the critical values for small $\Delta / t$ :

$$
U_{c 1}^{0}=\frac{2 \pi t}{\ln (t / \Delta)}\left[1-\frac{C}{\ln (t / \Delta)}+\cdots\right]
$$

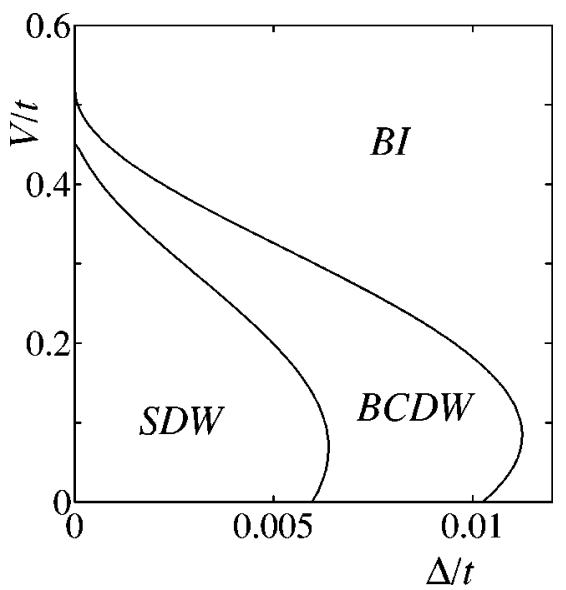

FIG. 12. Phase diagram of the half-filled extended ionic Hubbard model on the plane of $\Delta / t$ and $V / t$, where $U / t=1$.

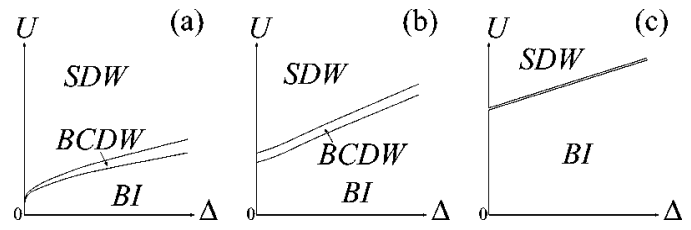

FIG. 13. Schematic phase diagram of the half-filled extended Hubbard model at (a) $V=0$, (b) $V \ll t$, and (c) $V \gg t$. The single lines represent second-order transitions, and the double line in (c) represents a first-order transition.

$$
U_{c 2}^{0}=\frac{2 \pi t}{\ln (t / \Delta)}\left[1+C^{\prime} \frac{\ln [\ln (t / \Delta)]}{\ln (t / \Delta)}+O\left(\frac{1}{\ln (t / \Delta)}\right)\right],
$$

where $C$ and $C^{\prime}$ are positive constants of order unity. The $\Delta$ dependence of $U_{c 1}^{0}$ is different from the result in Refs. 32 since the lowest correction to $2 \pi t / \ln (t / \Delta)$ is not $O(\ln [\ln (t / \Delta)] / \ln (t / \Delta))$, but $O(1 / \ln (t / \Delta))$. Our results suggest that the ratio of $U_{c 2}^{0}$ to $U_{c 1}^{0}$ becomes $U_{c 2}^{0} / U_{c 1}^{0}=1$ $+C^{\prime} \ln [\ln (t / \Delta)] / \ln (t / \Delta)$. At present we do not know where this difference comes from. We extend this analysis to the case with finite $V(\ll U)$ and examine the $V$ dependence of $U_{c 1}$ and $U_{c 2}$. We note that $G_{\rho}(l) \neq G_{c}(l)$ in this case since the $\mathrm{SU}(2)$ symmetry of the charge sector is broken. We integrate the RG equations analytically for small $V \neq 0$ and obtain the corrections to order $V$,

$$
\begin{gathered}
U_{c 1}=U_{c 1}^{0}-V\left[\frac{2}{3}+O\left(\frac{1}{\ln (t / \Delta)}\right)\right], \\
U_{c 2}=U_{c 2}^{0}-V\left[\frac{2}{3}+O\left(\frac{\ln \ln (t / \Delta)}{\ln (t / \Delta)}\right)\right],
\end{gathered}
$$

implying that the BCDW state survives upon inclusion of the $V(\ll U)$ term. We note that $U_{c 1}$ and $U_{c 2}$ have a similar linear dependence on $V$. From Eqs. (4.23) and Figs. 11 and 12, we conclude that the phase diagram exhibits reentrant behavior as $V$ increases from zero with $\Delta$ and $U$ being fixed at values near a quantum critical point.

Since the Hamiltonian $H^{\prime}$ has three free parameters $(U / t$, $V / t$, and $\Delta / t$ ) at half filling, the ground-state phase diagram becomes a three-dimensional (3D) diagram. Instead of drawing such a 3D plot, here we show two-dimensional tomographic phase diagrams. Figure 13 shows schematic phase diagrams in the $\Delta-U$ plane for three typical cases $V / t=0, V / t$ $\ll 1$, and $V / t \gg 1$. We see that the nearest-neighbor repulsion enhances the BI phase and destroys the BCDW phase at large $V$, where the direct transition between the $\mathrm{BI}$ and SDW phases is first order. The recent numerical study of the ionic Hubbard model ${ }^{40}$ reports a similar phase diagram as Fig. 13(a). The first-order transition line in Fig. 13(c) asymptotically approaches the line $U=2 \Delta+2 \mathrm{~V}$.

Figure 14 shows schematic phase diagrams in the $\Delta-V$ plane for $U / t \ll 1$ and $U / t \gg 1$. At large $U$ and $V$ there appears a direct first-order transition between the BI and SDW phases in Fig. 14(b). This first-order transition is in agreement with the results obtained from the strong-coupling analysis ${ }^{47}$ and numerical calculations. ${ }^{48,62}$ 

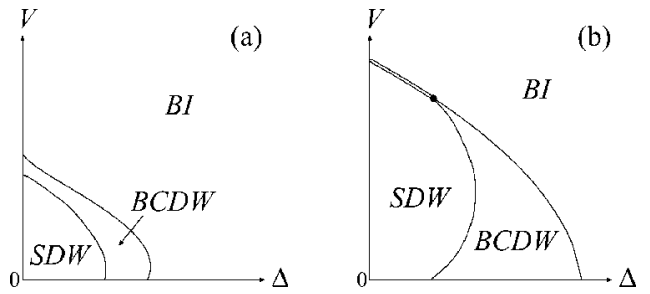

FIG. 14. Schematic phase diagram of the half-filled extended Hubbard model at (a) $U \ll t$ and (b) $U \gg t$. The single lines represent second-order transitions, and the double line represents a first-order transition.

\section{Discussions on previous numerical results}

As mentioned in Introduction, many groups have already reported on numerical studies of the ground-state phase diagram of the ionic Hubbard model. Various numerical techniques were used in these studies, including the densitymatrix renormalization-group (DMRG) method, ${ }^{34-37,45}$ the quantum Monte Carlo method, ${ }^{39,41}$ a finite-size cluster method, ${ }^{38}$ and a level crossing analysis. ${ }^{40}$ The main issue here is whether or not the SDI phase (BCDW phase) exists, and so far these numerical studies do not seem to have reached complete agreement yet. Although most of recent studies report that the SDI phase appears near the boundary between the SDW phase and the BI phase, ${ }^{35-37,39-41,45}$ there are still some conflicting claims in the literature. A less controversial issue ${ }^{63}$ is the determination of the second critical value $U_{c 2}$ at which a spin gap closes and which can be estimated by computing the spin gap directly ${ }^{34,35}$ or by examining the BCDW order parameter. ${ }^{37,45}$ The determination of the critical point $U_{c 1}$ and the critical behaviors around it are more controversial issues. One way to estimate the critical value $U_{c 1}$ is to use the complex parameter introduced by Resta and Sorrela. ${ }^{50}$ Its diverging behavior at $U=U_{c 1}$ indeed allows one to determine the critical point. ${ }^{34,39}$ Another way to determine the critical point is to find a gap closing point in excitation spectra. Since the charge sector is responsible for the quantum phase transition at $U=U_{c 1}$, one might try to look at a charge gap directly. However, numerical studies have found that a naive charge gap does not vanish at the critical point and is always finite. Recent studies have shown $n^{35,36,45}$ that the excitation gap that vanishes at $U$ $=U_{c 1}$ is the gap to the first excited state that has the same charge and spin quantum numbers as the ground state. Let us discuss this point in more detail below.

In numerical studies, ${ }^{34-36}$ the "charge gap" $\Delta_{c}$ was defined as $\Delta_{c}=E_{0}(L / 2+1, L / 2)+E_{0}(L / 2-1, L / 2)$ $-2 E_{0}(L / 2, L / 2)$, where $E_{0}\left(N_{\uparrow}, N_{\downarrow}\right)$ is the lowest energy of a finite-size system with an even number of sites $L$ that has $N_{\uparrow}$ up-spin and $N_{\downarrow}$ down-spin electrons. This quantity $\Delta_{c}$ measures the energy of the excitation with the topological charge $Q= \pm 1$ and $S_{z}= \pm 1 / 2$ [Eq. (4.7)], and is rather a singleelectron excitation gap. According to the bosonization theory (Sec. IV A), the charge transition at $U=U_{c 1}$ is described by the " $\varphi$ " theory and is in the Ising universality class. The transition occurs when two degenerate local minima of the effective potential for the charge fields merge into a single local minimum. As one approaches the transition point from the Ising ordered phase (that is, the SDI phase), the topological charge $Q= \pm 2 \alpha_{\theta} / \pi$ of a lowest-energy excitation is decreasing to zero, while excitations with $Q=1$ remain massive. Therefore the charge gap $\Delta_{c}$ does not vanish at this Ising critical point, and this quantum phase transition cannot be detected with $\Delta_{c}$. Qin et al. and Manmana et al. also used $\Delta_{e}=E_{1}(L / 2, L / 2)-E_{0}(L / 2, L / 2)$ in their numerical analysis, where $E_{1}\left(N_{\uparrow}, N_{\downarrow}\right)$ is the energy of the first excited state. $^{35,45}$ The quantity $\Delta_{e}$ measures excited states with the same number of electrons, whose total topological charge $Q=0$ in the sine-Gordon scheme. In the Ising ordered phase, the first excited state with the topological charge $Q=0$ would be a bound state (or breather) of a soliton with the topological charge $+2 \alpha_{\theta} / \pi$ and an antisoliton with the charge $-2 \alpha_{\theta} / \pi$, whose energy vanishes at the critical point. On the other hand, in the Ising disordered phase near the critical point, the potential is almost flat and has very small curvature. The low-energy excitations would then be small oscillations around potential minima (rather than soliton/ antisoliton) whose energy approaches zero as $U \rightarrow U_{c 1}-0$. Thus the exciton gap $\Delta_{e}$ is a right measure to detect the quantum phase transition at $U=U_{c 1}$.

\section{EFFECT OF BOND DIMERIZATION}

In this section, we consider the 1D EHM with staggered bond dimerization, ${ }^{64,65}$ i.e., the Peierls modulation of the hopping matrix element. The total Hamiltonian $H^{\prime \prime}$ is given by $H^{\prime \prime}=H+H_{\delta}$, where $H$ is defined in Eq. (2.1) and

$$
H_{\delta}=\delta \sum_{j, \sigma}(-1)^{j}\left(c_{j, \sigma}^{\dagger} c_{j+1, \sigma}+\text { H.c. }\right) \text {. }
$$

Without loss of generality we can assume $\delta>0$. When $V$ $=0$, the model is called "Peierls-Hubbard model." The onedimensional Mott insulator, realized when $U>0$ and $V=0$, is known to be unstable against the Peierls distortion, ${ }^{1,7}$ and as a result the ground state changes from the SDW state into the BCDW state regardless of the magnitude of the Hubbard interaction $U$. Such an instability comes from the fact that the bond dimerization tends to concentrate the electron density onto bonds, without any conflict with the Hubbard, $U$, repulsion. ${ }^{36}$ However, the nearest-neighbor Coulomb repulsion $V$ competes with this $\delta$ term, since the $V$ interaction likes to localize two electrons on a single site and promotes the CDW state. Here we investigate the instability of the BCDW state against the intersite Coulomb repulsion $V$, and clarify the critical behavior near the transition between the BCDW state and the CDW state.

The bond dimerization $H_{\delta}$ is bosonized as $H_{\delta}=\int d x \mathcal{H}_{\delta}$, where

$$
\mathcal{H}_{\delta}=-\frac{g_{\delta}}{2(\pi a)^{2}} \cos \theta \cos \phi
$$

and $g_{\delta}=8 \pi \delta a$. One finds that the EHM with the bond dimerization also has a two-component DSG structure. Here the charge phase field $\theta$ is subjected to the potential $\cos \theta$ 
TABLE III. Possible ground states and the position of locked phase fields, determined from Eq. (5.4).

\begin{tabular}{lc}
\hline \hline \multicolumn{1}{c}{ Phase } & $(\theta, \phi)$ \\
\hline SDW & $\left(0, \pm \gamma_{\phi}\right),\left(\pi, \pm\left(\pi-\gamma_{\phi}\right)\right)$ \\
CDW & $\left( \pm \gamma_{\theta}, 0\right),\left( \pm\left(\pi-\gamma_{\theta}\right), \pi\right)$ \\
PI (for $\left.g_{\delta}>0\right)$ & $(0,0),(\pi, \pi)$ \\
PI (for $\left.g_{\delta}<0\right)$ & $(0, \pi),(\pi, 0)$ \\
BSDW & $( \pm \pi / 2, \pm \pi / 2)$ \\
\hline \hline
\end{tabular}

instead of $\sin \theta$ of the $g_{\Delta}$ term [Eq. (4.2)], while the locking potential for the spin phase field $\phi$ has the same structure as that of the $g_{\Delta}$ term.

It is important to note that the BCDW order parameter $\mathcal{O}_{\text {BCDW }}$ takes a nonvanishing expectation value for any $U$ and $V$ if $\delta \neq 0$, as $\mathcal{H}_{\delta} \propto \mathcal{O}_{\mathrm{BCDW}}$. In this section we will not use the term BCDW to characterize phases, and, in particular, the phase containing the trivial Peierls insulator $(U=V=0$ and $\delta \neq 0$ ) is called the Peierls insulating (PI) phase.

\section{A. Semiclassical analysis}

We begin with semiclassical analysis of the model with the $g_{\delta}$ term. We neglect spatial variations of the phase fields in $\mathcal{H}+\mathcal{H}_{\delta}$ and consider the potential

$$
\begin{aligned}
V_{\delta}(\theta, \phi)= & -g_{c} \cos 2 \theta+g_{s} \cos 2 \phi-g_{c s} \cos 2 \theta \cos 2 \phi \\
& -g_{\delta} \cos \theta \cos \phi,
\end{aligned}
$$

where $g_{c s}=g_{3 \|}<0$.

First, we consider the simpler case where $g_{c s}=0$, which corresponds to the situation where $g_{c s}$ is irrelevant in the RG sense. The potential in this case is

$$
V_{\delta}^{0}(\theta, \phi)=-g_{c} \cos 2 \theta+g_{s} \cos 2 \phi-g_{\delta} \cos \theta \cos \phi .
$$

The positions of the potential minima are determined by the saddle-point equations $\partial V_{\delta}^{0}(\theta, \phi) / \partial \theta=0$ and $\partial V_{\delta}^{0}(\theta, \phi) / \partial \phi$ $=0$. We find that the potential has the double-well structure for the $\theta(\phi)$ phase field when $g_{c}<-\left|g_{\delta}\right| / 4\left(g_{s}>\left|g_{\delta}\right| / 4\right)$. Here we introduce $\gamma_{\theta}^{0}$ and $\gamma_{\phi}^{0}\left(0 \leqslant \gamma_{\theta}^{0}, \gamma_{\phi}^{0} \leqslant \pi\right)$ defined by

$$
\gamma_{\theta}^{0}=\left|\cos ^{-1}\left(-\frac{g_{\delta}}{4 g_{c}}\right)\right|, \quad \gamma_{\phi}^{0}=\left|\cos ^{-1}\left(\frac{g_{\delta}}{4 g_{s}}\right)\right|
$$

for $\left|g_{\delta} / g_{c}\right| \leqslant 4$ and $\left|g_{\delta} / g_{c}\right| \leqslant 4$, respectively. The solutions to the saddle-point equations can be classified into the following four classes: (i) the PI state, $(\theta, \phi)$ $=(0,0),(0, \pi),(\pi, 0)$, or $(\pi, \pi)\left[\right.$ for $g_{\delta}>0$, the phase fields are locked at $(\theta, \phi)=(0,0)$ or $(\pi, \pi)$, while for $g_{\delta}<0$ the phase fields are locked as $(\theta, \phi)=(0, \pi)$ or $(\pi, 0)]$; (ii) the pure BSDW state, $(\theta, \phi)=(\pi / 2, \pm \pi / 2)$ or $(-\pi / 2, \pm \pi / 2)$; (iii) the "SDW" state with both the SDW order and the BCDW order, $(\theta, \phi)=\left(0, \pm \gamma_{\phi}^{0}\right)$ or $\left(\pi, \pm\left(\pi-\gamma_{\phi}^{0}\right)\right)$; and (iv) finally, the "CDW" state with both the CDW order and the BCDW order, $(\theta, \phi)=\left( \pm \gamma_{\theta}^{0}, 0\right)$ or $\left( \pm\left(\pi-\gamma_{\theta}^{0}\right), \pi\right)$. The

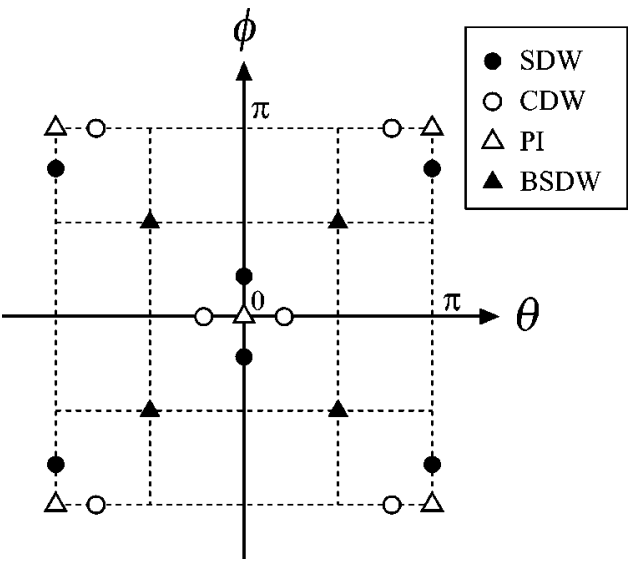

FIG. 15. Positions of locked phase fields $\theta$ and $\phi$ in the respective states for $g_{\delta}>0$.

possible ground states and positions of locked phase fields are summarized in Table III and Fig. 15. In these states the potential energy reads

$$
\begin{gathered}
V_{\mathrm{PI}}^{0}=-g_{c}+g_{s}-\left|g_{\delta}\right|, \\
V_{\mathrm{BSDW}}^{0}=+g_{c}-g_{s}, \\
V_{\mathrm{SDW}}^{0}=-g_{c}-g_{s}-\frac{g_{\delta}^{2}}{8 g_{s}}, \\
V_{\mathrm{CDW}}^{0}=+g_{c}+g_{s}+\frac{g_{\delta}^{2}}{8 g_{c}} .
\end{gathered}
$$

In deriving Eqs. (5.6c) and (5.6d), we have assumed $\left|g_{\delta} / g_{s}\right| \leqslant 4$ and $\left|g_{\delta} / g_{c}\right| \leqslant 4$, respectively. The PI state is stabilized by the first-order contribution of the $g_{\delta}$ term. Furthermore, if $g_{s}>0\left(g_{c}<0\right)$, the SDW state (the CDW state) is also stabilized due to second-order contribution of $g_{\delta}$. The phase diagram obtained by comparing these energies is shown in Fig. 16.

From the above semiclassical analysis one might conclude that the topological charge $S_{z}$ [Eq. (4.7)] becomes fractional in the SDW phase and that the Ising-type phase transition in the spin sector takes place on the boundary between the PI state and the SDW state. However, as discussed in Sec. IV, the global SU(2) symmetry prohibits the Ising criticality in the spin sector and changes the SDW phase in Fig. 16 into the PI phase.

Next we include the $g_{c s}$ term. Table III still stands if we replace $g_{c}$ and $g_{s}$ with $g_{c}-\left|g_{c s}\right|$ and $g_{s}+\left|g_{c s}\right|$ in $\gamma_{\theta}^{0}$ and $\gamma_{\phi}^{0}$, respectively. The phase diagram obtained by minimizing the potential energy $V_{\delta}(\theta, \phi)$ is shown in Fig. 17. New features compared with Fig. 16 are the appearance of a first-order transition line and of the new phase in which the ground state has the coexisting order of the SDW, CDW, BCDW, and BSDW. The new phase is shown as the shaded region in Fig. 17 , which is surrounded by the three curves defined by

$$
\left(g_{c}+\left|g_{c s}\right|\right)\left(g_{s}-\left|g_{c s}\right|\right)=-\frac{g_{\delta}^{2}}{16},
$$




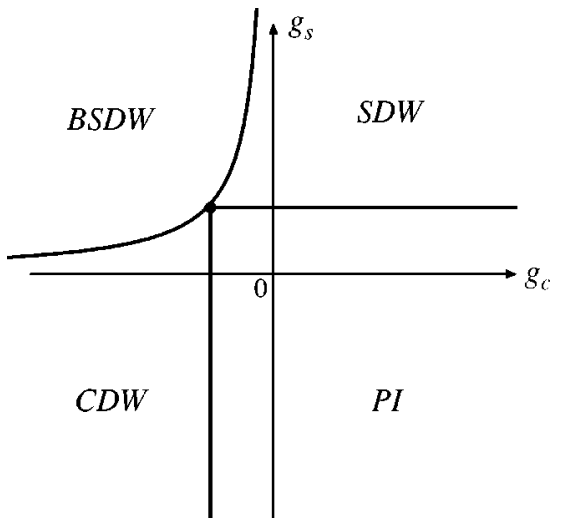

FIG. 16. Phase diagram obtained by minimizing the potential energy $V_{\delta}^{0}(\theta, \phi)$ [Eq. (5.4)]. The phase boundary of the BSDW state is given by the curve $g_{c} g_{s}=-g_{\delta}^{2} / 16$ with $g_{c}<0$. The phase boundary between the PI state and the SDW state and that between the PI state and the CDW state are given by the lines $g_{s}=\left|g_{\delta}\right| / 4$ with $g_{c}$ $>\left|g_{\delta}\right| / 4$ and $g_{c}=-\left|g_{\delta}\right| / 4$ with $g_{s}<\left|g_{\delta}\right| / 4$, respectively. All the phase transitions in this figure are continuous. A multicritical point is at $\left(g_{c}, g_{s}\right)=\left(-\left|g_{\delta}\right| / 4,\left|g_{\delta}\right| / 4\right)$.

$$
\begin{aligned}
& \left(g_{c}+\left|g_{c s}\right|\right)\left(g_{s}+\left|g_{c s}\right|\right)^{2}=-\frac{g_{\delta}^{2}}{16}\left(g_{s}-\left|g_{c s}\right|\right), \\
& \left(g_{s}-\left|g_{c s}\right|\right)\left(g_{c}-\left|g_{c s}\right|\right)^{2}=-\frac{g_{\delta}^{2}}{16}\left(g_{c}+\left|g_{c s}\right|\right) .
\end{aligned}
$$

Let us focus on the phases which can be realized when $g_{s} \simeq g_{c}$, in view of the fact that in the extended Hubbard model both $g_{s}\left(=g_{1 \perp}\right)$ and $g_{c}\left(=g_{3 \perp}\right)$ are given by $(U$ $-2 V$ ) in the lowest order. Along the line $g_{s} \simeq g_{c}$ in Figs. 16 and 17 , there are three possible phases: the SDW state, the PI

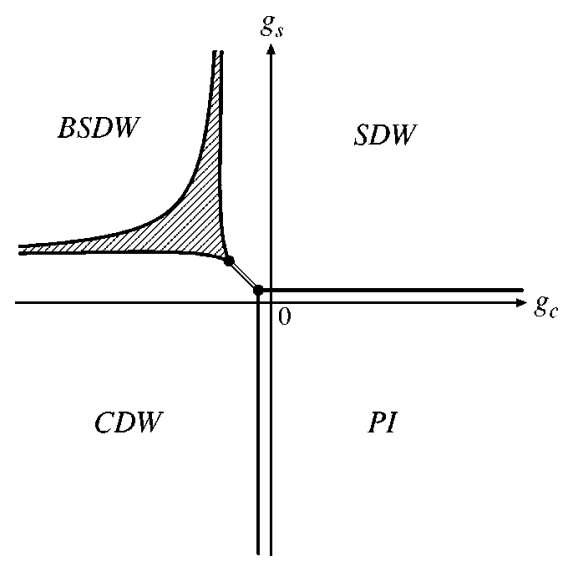

FIG. 17. Phase diagram obtained by minimizing the potential energy $V_{\delta}(\theta, \phi)$ [Eq. (5.3)] drawn for $\left|g_{\delta}\right| / 8<\left|g_{c s}\right|<\left|g_{\delta}\right| / 4$. Multicritical points are located at $\left(g_{c}, g_{s}\right)=\left(-\left|g_{c s}\right|,\left|g_{c s}\right|\right)$ and $\left(-\frac{1}{4}\left|g_{\delta}\right|\right.$ $\left.+\left|g_{c s}\right|, \frac{1}{4}\left|g_{\delta}\right|-\left|g_{c s}\right|\right)$. The boundary of the BSDW phase is $\left(g_{c}\right.$ $\left.+\left|g_{c s}\right|\right)\left(g_{s}-\left|g_{c s}\right|\right)<-g_{\delta}^{2} / 16$. The edges of the PI phase are defined by the lines $g_{c}=-\frac{1}{4}\left|g_{\delta}\right|+\left|g_{c s}\right|$ and $g_{s}=\frac{1}{4}\left|g_{\delta}\right|-\left|g_{c s}\right|$. The double line denotes a first-order transition, and the single lines denote continuous transitions. Within the semiclassical analysis the ground state in the shaded region has the coexisting order of the SDW, CDW, BCDW, and BSDW. state, and the CDW state. Since the SDW state is prohibited by the SU(2) symmetry and becomes the PI state, we expect to have only two phases, the PI state and the CDW state, and a single phase transition between them. The transition is continuous at $\left|g_{c s} / g_{\delta}\right| \ll 1$ and changes into a discontinuous transition when $g_{c s}$ exceeds $\left|g_{\delta}\right| / 4$.

\section{B. Renormalization-group analysis}

Next we perform perturbative RG analysis to take into account quantum fluctuations. The one-loop RG equations for coupling constants in $\mathcal{H}+\mathcal{H}_{\delta}$ are given by

$$
\begin{aligned}
& \frac{d}{d l} G_{\delta}=+G_{\delta}+\frac{1}{2} G_{\delta} G_{\rho}+G_{\delta} G_{c}-\frac{3}{2} G_{\delta} G_{s}+\frac{3}{4} G_{\delta} G_{c s} \\
&-\frac{3}{8} G_{\delta} G_{\rho s} \\
& \frac{d}{d l} G_{\rho}=+\frac{1}{4} G_{\delta}^{2}+2 G_{c}^{2}+G_{c s}^{2}+G_{s} G_{\rho s} \\
& \frac{d}{d l} G_{c}=+\frac{1}{4} G_{\delta}^{2}+2 G_{\rho} G_{c}-G_{s} G_{c s}-G_{c s} G_{\rho s}
\end{aligned}
$$

$$
\frac{d}{d l} G_{s}=-\frac{1}{4} G_{\delta}^{2}-2 G_{s}^{2}-G_{c} G_{c s}-G_{c s}^{2}
$$

$$
\begin{aligned}
\frac{d}{d l} G_{c s}= & +\frac{1}{4} G_{\delta}^{2}-2 G_{c s}+2 G_{\rho} G_{c s}-4 G_{s} G_{c s}-2 G_{c} G_{s} \\
& -2 G_{c} G_{\rho s}-4 G_{c s} G_{\rho s}
\end{aligned}
$$

$$
\begin{aligned}
\frac{d}{d l} G_{\rho s}= & -\frac{1}{4} G_{\delta}^{2}-2 G_{\rho s}+2 G_{\rho} G_{s}-4 G_{c} G_{c s} \\
& -4 G_{c s}^{2}-4 G_{s} G_{\rho s} .
\end{aligned}
$$

The initial value of $G_{\delta}(l)$ is given by $G_{\delta}(0)=2 \delta / t$ and those of the other coupling constants are $G_{\nu}(0)$ $=g_{\nu} /(4 \pi t)$. We note that these RG equations are invariant under the sign change of $G_{\delta}(l)$. We can thus assume $G_{\delta}(0) \geqslant 0$ without losing generality.

To find the ground-state phase diagram of the system, we solve the scaling equations (5.8)-(5.13) numerically, as in the preceding sections. We determine to which phase the ground state belongs by looking at which one of the couplings $G_{\delta}(l), G_{c}(l), G_{s}(l)$, and $G_{c s}(l)$ becomes most relevant. For repulsive $U$ and $V$ there are four possibilities as listed below.

(i) If $G_{c}$ is most relevant and $G_{c}(l) \rightarrow 1$ at $l=l_{\rho+}$, then the phase field $\theta$ is locked at $\theta=0$ or $\pi$, and the effective Hamiltonian for the spin sector at $l \geqslant l_{\rho+}$ becomes 


$$
\begin{aligned}
\mathcal{H}_{\sigma+}^{\mathrm{eff}}= & \frac{v_{F}}{2 \pi} \sum_{p= \pm}\left(\partial_{x} \phi_{p}\right)^{2}-\frac{v_{F}}{\pi} G_{s}^{*}\left(\partial_{x} \phi_{+}\right)\left(\partial_{x} \phi_{-}\right) \\
& \mp \frac{v_{F}}{\pi a^{2}} G_{\delta}^{*} \cos \phi+\frac{v_{F}}{\pi a^{2}} G_{s}^{*} \cos 2 \phi
\end{aligned}
$$

where $G_{s}^{*}=G_{s}\left(l_{\rho+}\right)-G_{c s}\left(l_{\rho+}\right)$ and $G_{\delta}^{*}=G_{\delta}\left(l_{\rho+}\right)$, and the sign $-I+$ of the $G_{\delta}^{*}$ term corresponds to the location of the phase locking $\theta=0 / \pi$. This effective theory is the same as Eq. (4.19). As seen before, regardless of the sign of $G_{s}^{*}$, the phase field $\phi$ is locked at $\phi=0$ or $\pi$ depending on the position of the charge phase locking $\theta=0$ or $\pi$. Thus we have the phase locking $(\theta, \phi)=(0,0)$ or $(\pi, \pi)$, i.e., the PI state as the ground state. We note that due to the $\mathrm{SU}(2)$ spin rotation symmetry the SDW state cannot be realized even if $G_{s}^{*}$ $>0$.

(ii) If $G_{c}$ is most relevant and $G_{c}(l) \rightarrow-1$ at $l=l_{\rho-}$, then the phase field $\theta$ is locked at $\theta= \pm \pi / 2$. The effective Hamiltonian for the spin part is

$$
\begin{aligned}
\mathcal{H}_{\sigma-}^{\mathrm{eff}}= & \frac{v_{F}}{2 \pi} \sum_{p}\left(\partial_{x} \phi_{p}\right)^{2}-\frac{v_{F}}{\pi} G_{s}^{*}\left(\partial_{x} \phi_{+}\right)\left(\partial_{x} \phi_{-}\right) \\
& +\frac{v_{F}}{\pi a^{2}} G_{s}^{*} \cos 2 \phi
\end{aligned}
$$

where $G_{s}^{*}=G_{s}\left(l_{\rho_{-}}\right)+G_{c s}\left(l_{\rho_{-}}\right)$. We have verified numerically that $G_{s}^{*}$ always becomes negative in this case. The $G_{s}^{*}$ terms are then marginally relevant $\left[G_{s}^{*}(l) \rightarrow-\infty\right.$ under scaling]. The phase fields are then locked at $(\theta, \phi)$ $=( \pm \pi / 2,0),( \pm \pi / 2, \pi)$, which corresponds to the CDW phase with $\gamma_{\theta} \rightarrow \pi / 2$ (i.e., $g_{\delta} \longrightarrow 0$, see Table III). Since $\mathcal{H}_{\delta}$ $\propto \mathcal{O}_{\text {BCDW }}$ the order parameter of the BCDW should have a nonvanishing expectation value. We thus conclude that the ground state is in the CDW phase.

(iii) If either $G_{\delta}$ or $G_{c s}$ is most relevant, both charge and spin fluctuations are suppressed. In this case the semiclassical treatment is justified, and we can determine to which phase the ground state belongs by substituting $G_{c}$ and $G_{s}$ to $g_{c}$ and $g_{s}$ in Fig. 17.

(iv) If $G_{s}$ is most relevant and $G_{s}(l) \rightarrow-1$ at $l=l_{\sigma}$, the spin fluctuations are suppressed and the phase field $\phi$ is locked at $\phi \rightarrow 0$ or $\pi$ below this energy scale. The effective Hamiltonian at $l \geqslant l_{\sigma}$ is given by

$$
\begin{aligned}
\mathcal{H}_{\rho}^{\mathrm{eff}}= & \frac{v_{F}}{2 \pi} \sum_{p= \pm}\left(\partial_{x} \theta_{p}\right)^{2}+\frac{v_{F}}{\pi} G_{\rho}^{*}\left(\partial_{x} \theta_{+}\right)\left(\partial_{x} \theta_{-}\right) \\
& \mp \frac{v_{F}}{\pi a^{2}} G_{\delta}^{*} \cos \theta-\frac{v_{F}}{\pi a^{2}} G_{c}^{*} \cos 2 \theta
\end{aligned}
$$

where $G_{\rho}^{*}=G_{\rho}\left(l_{\sigma}\right)-G_{\rho s}\left(l_{\sigma}\right), G_{c}^{*}=G_{c}\left(l_{\sigma}\right)+G_{c s}\left(l_{\sigma}\right)$, and $G_{\delta}^{*}=G_{\delta}\left(l_{\sigma}\right)$. The sign $-/+$ of the $G_{\delta}^{*}$ term corresponds to the phase locking $\phi=0 / \pi$. Both of the nonlinear terms $\cos \theta$ and $\cos 2 \theta$ are relevant perturbations. If $G_{c}^{*}<0$, these two terms compete with each other, and this DSG model exhibits the Ising criticality. The ground state is either in the PI phase

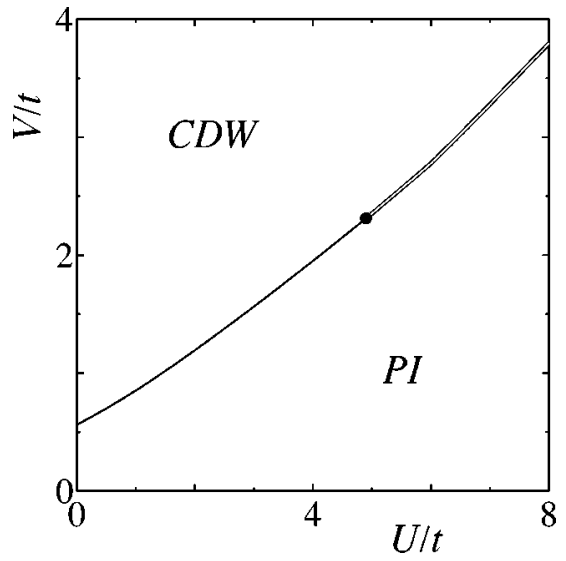

FIG. 18. Phase diagram of the half-filled extended Hubbard model with $\delta / t=0.1$. The second-order transition line (single line) turns into the first-order transition line (double line) at the tricritical point $\left(U_{c}, V_{c}\right) \approx(4.9 t, 2.3 t)$.

or in the CDW phase, and there is an Ising-type quantum phase transition between the two phases. Here we estimate the Ising critical point from the semiclassical analysis. That is, the critical value is determined from the condition $G_{c}^{*} / G_{\delta}^{*}=-1 / 4$ (see Fig. 16). If $G_{c}^{*}>0$, these two terms do not compete and thus the phase locking is $\theta=0(\pi)$ for $\phi$ $=0(\pi)$, where the ground state is the PI state.

The resultant phase diagram in the $U-V$ plane is shown in Fig. 18. In the weak-coupling region, the transition from the PI state to the CDW state is characterized by the appearance of the double-well structure of the effective potential to the $\theta$ field, and thus the phase transition in Fig. 18 belongs to the Ising universality class. As we increase $U$ and $V$, there appears a tricritical point at $\left(U_{c}, V_{c}\right) \approx(4.9 t, 2.3 t)$, where the phase transition changes from second order to first order.

Figure 19 shows schematic phase diagrams in the $\delta$ - $U$ plane for $V \ll t$ and $V / t \gg 1$. When $\delta=0$, we obtain three phases (the CDW, BCDW, and SDW phases) for $V \ll t$ (a) and two phases (the CDW and SDW phases) for $V \gg t$ (b), as we discussed in Sec. III (see Fig. 7). Upon turning on $\delta$, the SDW ground state changes into the PI state, where the transition is described by the Gaussian theory. On the other hand, the BCDW state changes into the PI state without accompanying any singularity: This change is merely lifting of the doubly degenerate BCDW ground states.

Figure 20 shows schematic phase diagrams in the $\delta$ - $V$ plane for $U=0, U / t \ll 1$, and $U / t \gg 1$. At $U=0$ we have a

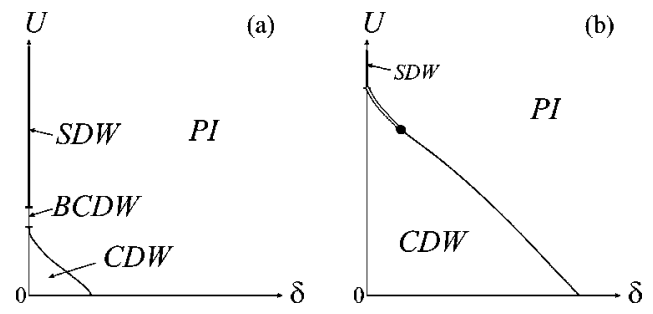

FIG. 19. Schematic phase diagram of the half-filled extended Hubbard model at (a) $V \ll t$ and (b) $V \gg t$. The single lines represent second-order transitions, and the double line represents a first-order transition. 

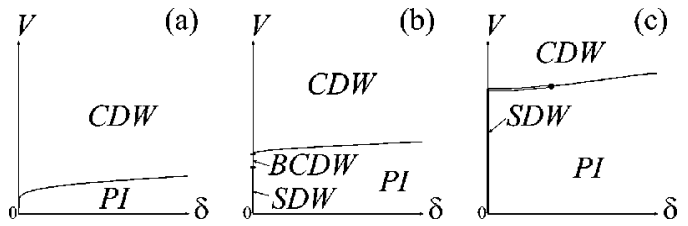

FIG. 20. Schematic phase diagram of the half-filled extended Hubbard model at (a) $U=0$, (b) $U \ll t$, and (c) $U \gg t$. The single lines represent second-order transitions, and the double line represents a first-order transition.

single critical value $V_{c}$ which has the $\delta$ dependence given by $V_{c} \propto 1 / \ln (t / \delta)$ for small $\delta$. As $U$ and $V$ increase, the phase boundary approaches the $U=2 V$ line. The asymptotic form of $V_{c}$ for $U, V \gg \delta$ and $\delta \ll t$ is given by $V_{c}=\frac{1}{2} U$ $+C^{\prime \prime} U(\delta / t)^{2 U / \pi t}$, where $C^{\prime \prime}$ is a numerical constant of the order of unity (see also Fig. 18).

\section{CONCLUSIONS}

In this paper we have studied the ground-state phase diagram of the one-dimensional extended Hubbard model with on-site and nearest-neighbor repulsion $U$ and $V$. By including higher-order corrections to coupling constants in the $g$-ology, we have given a plausible theoretical argument within the RG approach for the mechanism of the appearance of the BCDW phase at $U \approx 2 V$ in the weak-coupling limit. Our two-step RG approach, however, is not complete in that there remains a weak cutoff dependence in the phase boundaries. This, albeit minor, defect should be resolved with use of a more sophisticated systematic RG procedure. Away from the weak-coupling limit the umklapp scattering between the parallel-spin electrons $g_{3 \|}$ tends to destabilize the BCDW state and eventually gives rise to a bicritical point where the two continuous-transition lines merge into the SDW-CDW first-order transition line (Fig. 7). We should note, however, that there still remains a difficult question as to whether our phase diagram is qualitatively correct near the multicritical point (which we call bicritical). One could imagine, for example, a possibility that a continuous phase transition between the BCDW state and the CDW state becomes first order before reaching the multicritical point, due to higherorder effects that are ignored in our analysis. If the correct topology of the phase diagram is indeed the same as ours (Fig. 7), then the critical properties of the multicritical point remain to be understood. We hope that these issues will be resolved by future studies.

We have also examined effects of additional staggered site potential and bond dimerization in the extended Hubbard model. In the presence of the staggered site potential, we have found that the BCDW state is smoothly connected to the SDI phase which is obtained for $V=0$ by Fabrizio et al ${ }^{32}$ In this BCDW phase the BCDW order coexists with the $\mathrm{CDW}$ order, and the quantum phase transition between the $\mathrm{BI}$ phase (or the CDW phase) and the BCDW phase belongs to the Ising universality class $\left(c=\frac{1}{2} \mathrm{CFT}\right)$. For finite $V$ the BCDW phase is also destabilized by the $g_{3 \|}$ term, and the direct first-order quantum phase transition between the SDW state (= Mott insulating state) and the BI state takes place
(Fig. 11). In the presence of the staggered bond dimerization the SDW phase becomes unstable and the ground state at $V=0$ turns out to be the Peierls insulating state. For $V \neq 0$ the phase diagram consists of two phases, the PI state and the CDW state, which are separated by a phase transition line of the Ising criticality (Fig. 18).

\section{ACKNOWLEDGMENTS}

One of the authors (M.T.) thanks E. Orignac, M. Sugiura, Y. Suzumura, K. Yonemitsu, and H. Yoshioka for valuable discussions. The authors also thank S. Qin for useful discussions. A.F. is grateful to Aspen Center for Physics for its hospitality, where this paper was finished. This work was supported in part by a Grant-in-Aid for Scientific Research on Priority Areas from the Ministry of Education, Culture, Sports, Science and Technology, Japan (Grant No. 12046238).

\section{APPENDIX A: BOSONIZATION}

In this section, we derive the phase Hamiltonian of the 1D extended Hubbard model by using the Abelian bosonization method. ${ }^{66}$ We include not only the marginal terms but the leading irrelevant terms which play a crucial role in the firstorder SDW-CDW transition at strong coupling.

The Lagrangian for the free massless boson theory in a two-dimensional Euclidean space is given by

$$
L_{\theta}=\frac{1}{4 \pi} \int d x\left[v\left(\partial_{x} \theta\right)^{2}+\frac{1}{v}\left(\partial_{\tau} \theta\right)^{2}\right],
$$

where $\theta$ is a bosonic field, $\tau$ is the imaginary time, and $v$ is velocity. The variable canonically conjugate to $\theta$ is given by

$$
\Pi \equiv \frac{\partial L}{\partial \dot{\theta}}=\frac{i}{2 \pi v} \partial_{\tau} \theta
$$

where $\dot{\theta}=\partial \theta / \partial t$ and $t$ is the real time $(\tau=i t)$. As usual this system is quantized by imposing the commutation relation at equal times: $\left[\theta(x), \Pi\left(x^{\prime}\right)\right]=i \delta\left(x-x^{\prime}\right)$. Thus the Hamiltonian for the free boson theory is given by $H_{\theta}$ $=i \int d x \Pi \partial_{\tau} \theta+L_{\theta}$, i.e.,

$$
H_{\theta}=\frac{v}{4 \pi} \int d x\left[(2 \pi \Pi)^{2}+\left(\frac{d \theta}{d x}\right)^{2}\right] .
$$

Introducing two copies of this theory with fields $\theta$ and $\phi$ and velocity $v=v_{F}$, we arrive at $H_{0}$ [Eq. (2.11)], where the fields $\theta$ and $\phi$ represent the "charge" and "spin" degrees of freedom. The chiral bosonic fields $\theta_{ \pm}(x, \tau)$ and $\phi_{ \pm}(x, \tau)$ are introduced in Eqs. (2.12) and (2.13), respectively, where the right-moving (left-moving) fields are functions of $\tau$ $-i\left(x / v_{F}\right)\left[\tau+i\left(x / v_{F}\right)\right] .{ }^{66}$ The phase field $\theta(\phi)$ and its dual phase field $\widetilde{\theta}(\widetilde{\phi})$ are written in terms of the chiral fields as

$$
\begin{array}{cc}
\theta=\theta_{+}+\theta_{-}, & \widetilde{\theta}=\theta_{+}-\theta_{-}, \\
\phi=\phi_{+}+\phi_{-}, & \widetilde{\phi}=\phi_{+}-\phi_{-} .
\end{array}
$$


They satisfy the following commutation relations:

$$
\left[\theta(x), \widetilde{\theta}\left(x^{\prime}\right)\right]=\left[\phi(x), \widetilde{\phi}\left(x^{\prime}\right)\right]=-i 2 \pi \Theta\left(-x+x^{\prime}\right),
$$

where $\Theta(x)$ is the Heaviside step function.

The electron field operators $\psi_{p, \sigma}(x)$ are given in Eq. (2.17) in terms of a new set of chiral bosonic fields $\varphi_{p, \sigma}$ introduced in Eq. (2.15). In this bosonization scheme $\psi_{+, \sigma}$ and $\psi_{-, \sigma}$ anticommute, and we only need to introduce the Klein factor $\kappa_{\sigma}$ to ensure the anticommutation relation between fields with different spins; cf. the so-called constructive bosonization method. ${ }^{67}$ From Eqs. (2.16) and (2.17) the electron-density operator becomes

$$
\rho_{p, \sigma}(x) \equiv: \psi_{p, \sigma}^{\dagger} \psi_{p, \sigma}:=\frac{1}{2 \pi} \frac{d}{d x} \varphi_{p, \sigma}(x) .
$$

As is well known, the Hamiltonian density of free bosons (2.11), i.e.,

$$
\mathcal{H}_{0}=\frac{v_{F}}{4 \pi} \sum_{p= \pm} \sum_{\sigma}\left(\frac{d \varphi_{p, \sigma}}{d x}\right)^{2}=\pi v_{F} \sum_{p, \sigma} \rho_{p, \sigma}^{2}(x),
$$

is equivalent to the Hamiltonian density of free fermions with linear energy dispersion, Eq. (2.9). This can be shown, for example, by using the OPE method. ${ }^{68}$

Next we bosonize the interaction term $H_{\text {int }}$. Without the nearest-neighbor repulsion $V$, this can be easily done as ${ }^{18,54}$

$$
\begin{aligned}
\mathcal{H}_{\mathrm{int}}^{V=0}= & \frac{g_{4 \|}+g_{4 \perp}}{4 \pi^{2}}\left[\left(\partial_{x} \theta_{+}\right)^{2}+\left(\partial_{x} \theta_{-}\right)^{2}\right]+\frac{g_{4 \|}-g_{4 \perp}}{4 \pi^{2}}\left[\left(\partial_{x} \phi_{+}\right)^{2}\right. \\
& \left.+\left(\partial_{x} \phi_{-}\right)^{2}\right]+\frac{g_{2 \|}+g_{2 \perp}-g_{1 \|}}{2 \pi^{2}}\left(\partial_{x} \theta_{+}\right)\left(\partial_{x} \theta_{-}\right) \\
& +\frac{g_{2 \|}-g_{2 \perp}-g_{1 \|}}{2 \pi^{2}}\left(\partial_{x} \phi_{+}\right)\left(\partial_{x} \phi_{-}\right)-\frac{g_{3 \perp}}{2(\pi a)^{2}} \cos 2 \theta \\
& +\frac{g_{1 \perp}}{2(\pi a)^{2}} \cos 2 \phi,
\end{aligned}
$$

where $g$ 's are given in and below Eq. (2.8). In the presence of $V$, the matrix element of the umklapp process with parallel spins $H_{g_{3 \|}}$ [the $g_{3 \|}$ process in Eq. (2.4)] has a finite amplitude at lowest order in $g$-ology. This term can be bosonized as

$$
\mathcal{H}_{g_{3 \|}}=-\frac{g_{3 \|}}{2(\pi a)^{2}} \cos 2 \theta \cos 2 \phi,
$$

where $g_{3 \|}=-2 V a$ in the lowest order in $V$. This term, which couples the charge and spin degrees of freedom, is often neglected since it is an irrelevant perturbation with scaling dimension 4, consisting of $\operatorname{dim}[\cos 2 \theta]=2$ plus $\operatorname{dim}[\cos 2 \phi]=2$. Cannon and Fradkin were the first to suggest that this term should play an important role in the firstorder SDW-CDW transition in the half-filled EHM. ${ }^{22}$ Voit then derived RG equations including this term. However he did not include all the operators with scaling dimension 4 and failed to keep the spin-rotational SU(2) symmetry. ${ }^{24} \mathrm{We}$ have to be careful in dealing with the $V$ interaction to include the important terms with scaling dimension up to 4 . To this end, we focus on the $V$ interaction and bosonize each scattering process separately.

First, the $g_{1 \|}$ term ${ }^{54}$ representing the backward scattering with parallel spins is bosonized by using Eq. (2.17) as

$$
\begin{aligned}
V a \sum_{p, \sigma} & \psi_{p, \sigma}^{\dagger}(x) \psi_{-p, \sigma}(x) \psi_{-p, \sigma}^{\dagger}(x+a) \psi_{p, \sigma}(x+a) \\
= & -\frac{V a}{(2 \pi a)^{2}} \sum_{p, s= \pm} e^{i p[\theta(x+a)-\theta(x)]+i p s[\phi(x+a)-\phi(x)]} \\
= & \frac{V a}{2 \pi^{2}}\left[\sum_{p}\left(\partial_{x} \theta_{p}\right)^{2}+2\left(\partial_{x} \theta_{+}\right)\left(\partial_{x} \theta_{-}\right)\right] \\
& +\frac{V a}{2 \pi^{2}}\left[\sum_{p}\left(\partial_{x} \phi_{p}\right)^{2}+2\left(\partial_{x} \phi_{+}\right)\left(\partial_{x} \phi_{-}\right)\right] \\
& -\frac{V a}{4 \pi^{2}} a^{2}\left(\partial_{x} \theta\right)^{2}\left(\partial_{x} \phi\right)^{2}+\cdots,
\end{aligned}
$$

where we have expanded the exponent in the second line up to the order $a^{4}$ for the $\theta$ sector and the $\phi$ sector, separately. Since we are interested in operators that couple $\theta$ and $\phi$ as in Eq. (A10), we have discarded dimension- 4 terms such as $a^{4}\left(\partial_{x} \theta\right)^{4}$ and $a^{4}\left(\partial_{x} \phi\right)^{4}$ that involve only one sector. Such terms as $\left(\partial_{x} \theta_{+}\right)\left(\partial_{x} \theta_{-}\right)$and $\left(\partial_{x} \phi_{+}\right)\left(\partial_{x} \phi_{-}\right)$are already retained in Eq. (A9), while the last term proportional to $\left(\partial_{x} \theta\right)^{2}\left(\partial_{x} \phi\right)^{2}$ is a new term with scaling dimension $2+2$, which was missed in Ref. 24 . We note that the Fermi velocity is renormalized by the $g_{1 \|}$ term due to the presence of $\Sigma_{p}\left(\partial_{x} \theta_{p}\right)^{2}$ and $\Sigma_{p}\left(\partial_{x} \phi_{p}\right)^{2}$. This is in contrast with the conventional treatment where the velocity renormalization comes only from the forward scattering term $g_{4}{ }^{54}$

In a similar way, the interaction terms of backward and umklapp scattering with opposite spins (so-called $g_{1 \perp}$ and $g_{3 \perp}$ terms, ${ }^{54}$ respectively) are bosonized as

$$
\begin{aligned}
V a \sum_{p, \sigma} \psi_{p, \sigma}^{\dagger}(x) \psi_{-p, \sigma}(x) \psi_{-p, \bar{\sigma}}^{\dagger}(x+a) \psi_{p, \bar{\sigma}}(x+a) \\
=-\frac{V a}{(2 \pi a)^{2}} \sum_{p, s= \pm} e^{i p[\theta(x+a)-\theta(x)]-i p s[\phi(x+a)+\phi(x)]} \\
=-\frac{2 V a}{2(\pi a)^{2}} \cos 2 \phi+\frac{2 V a}{2 \pi^{2}}\left(\partial_{x} \theta_{+}\right)\left(\partial_{x} \theta_{-}\right) \cos 2 \phi \\
\quad+\frac{2 V a}{4 \pi^{2}}\left[\sum_{p}\left(\partial_{x} \theta_{p}\right)^{2}\right] \cos 2 \phi+\cdots,
\end{aligned}
$$




$$
\begin{aligned}
V a \sum_{p, \sigma} & \psi_{p, \sigma}^{\dagger}(x) \psi_{-p, \sigma}(x) \psi_{p, \bar{\sigma}}^{\dagger}(x+a) \psi_{-p, \bar{\sigma}}(x+a) \\
= & \frac{V a}{(2 \pi a)^{2}} \sum_{p, s= \pm} e^{-i p[\theta(x+a)+\theta(x)]+i p s[\phi(x+a)-\phi(x)]} \\
= & +\frac{2 V a}{2(\pi a)^{2}} \cos 2 \theta-\frac{2 V a}{2 \pi^{2}}\left(\partial_{x} \phi_{+}\right)\left(\partial_{x} \phi_{-}\right) \cos 2 \theta \\
& -\frac{2 V a}{4 \pi^{2}}\left[\sum_{p}\left(\partial_{x} \phi_{p}\right)^{2}\right] \cos 2 \theta+\cdots,
\end{aligned}
$$

where $\bar{\sigma}=\downarrow(\uparrow)$ for $\sigma=\uparrow(\downarrow)$. The potential $\cos 2 \phi$ in Eq. (A12) and the potential $\cos 2 \theta$ in Eq. (A13) are already retained in Eq. (A9), while the other terms are new and have the scaling dimension $2+2$.

The forward-scattering terms $\left(g_{2 \|}, g_{2 \perp}, g_{4 \|}\right.$, and $\left.g_{4 \perp}\right)$ do not generate operators of dimension $2+2$.

Hence the total Hamiltonian is given by

$$
\begin{aligned}
\mathcal{H}= & \frac{1}{2 \pi} \sum_{p}\left[v_{\rho}\left(\partial_{x} \theta_{p}\right)^{2}+v_{\sigma}\left(\partial_{x} \phi_{p}\right)^{2}\right]+\frac{g_{\rho}}{2 \pi^{2}}\left(\partial_{x} \theta_{+}\right)\left(\partial_{x} \theta_{-}\right) \\
& -\frac{g_{\sigma}}{2 \pi^{2}}\left(\partial_{x} \phi_{+}\right)\left(\partial_{x} \phi_{-}\right)-\frac{g_{3 \perp}}{2 \pi^{2} a^{2}} \cos 2 \theta+\frac{g_{1 \perp}}{2 \pi^{2} a^{2}} \cos 2 \phi \\
& +\frac{V a}{\pi^{2} a^{2}} \cos 2 \theta \cos 2 \phi+\frac{V a}{2 \pi^{2}}\left[\sum_{p}\left(\partial_{x} \theta_{p}\right)^{2}\right. \\
& \left.+2\left(\partial_{x} \theta_{+}\right)\left(\partial_{x} \theta_{-}\right)\right] \cos 2 \phi-\frac{V a}{2 \pi^{2}}\left[\sum_{p}\left(\partial_{x} \phi_{p}\right)^{2}\right. \\
& \left.+2\left(\partial_{x} \phi_{+}\right)\left(\partial_{x} \phi_{-}\right)\right] \cos 2 \theta-\frac{V a^{3}}{4 \pi^{2}}\left[\sum_{p}\left(\partial_{x} \theta_{p}\right)^{2}\right. \\
& \left.+2\left(\partial_{x} \theta_{+}\right)\left(\partial_{x} \theta_{-}\right)\right]\left[\sum_{p}\left(\partial_{x} \phi_{p}\right)^{2}+2\left(\partial_{x} \phi_{+}\right)\left(\partial_{x} \phi_{-}\right)\right] .
\end{aligned}
$$

The renormalized velocities are given by $v_{\rho}=2 t a+(U$ $+6 V) a /(2 \pi)$ and $v_{\sigma}=2 t a-(U-2 V) a /(2 \pi)$. The coupling constants $g_{1 \perp}$ and $g_{3 \perp}$ are defined in Eq. (2.8), and $g_{\rho}\left(\equiv g_{2 \|}+g_{2 \perp}-g_{1 \|}\right)$ and $g_{\sigma}\left(\equiv-g_{2 \|}+g_{2 \perp}+g_{1 \|}\right)$ are given by

$$
\begin{aligned}
& g_{\rho}=(U+6 V) a+\frac{C_{1}}{4 \pi t}(U-2 V)^{2} a+\frac{C_{2}}{\pi t} V^{2} a, \\
& g_{\sigma}=(U-2 V) a-\frac{C_{1}}{4 \pi t}(U-2 V)^{2} a-\frac{C_{2}}{\pi t} V^{2} a .
\end{aligned}
$$

For the discussion of the SDW-CDW transition in the 1D EHM, it is sufficient to have the coupling constants of dimension 4 in lowest order in $V$. We note that due to the $\mathrm{SU}(2)$ spin-rotation symmetry of the theory, the coupling constants for spin degrees of freedom must satisfy $g_{\sigma}$ $=g_{1 \perp}$, in any order of $U$ and $V$. To proceed further, we neglect the terms that involve $V \Sigma_{p}\left(\partial_{x} \theta_{p}\right)^{2}$ or $V \Sigma_{p}\left(\partial_{x} \phi_{p}\right)^{2}$ in Eq. (A14). These terms can lead to renormalization of the velocity through the RG transformation (see Appendix B). This effect can be ignored if we are interested in qualitative feature of the ground-state phase diagram of the model. The final form of the bosonized Hamiltonian is thus given by Eq. (2.19).

\section{APPENDIX B: DERIVATION OF RENORMALIZATION- GROUP EQUATIONS}

In this section, we derive one-loop RG equations for the coupling constants including those operators with higher scaling dimension. Our derivation is based on the operator product expansion (OPE) method. The interaction part of the action $S_{\mathrm{I}}$ in the presence of the staggered site potential $G_{\Delta}$ is given by

$$
\begin{aligned}
S_{\mathrm{I}}= & \frac{G_{\rho}}{\pi} \int d^{2} r\left(\partial_{z} \theta\right)\left(\partial_{z}^{-} \theta\right)-\frac{G_{\sigma}}{\pi} \int d^{2} r\left(\partial_{z} \phi\right)\left(\partial_{z}^{-} \phi\right) \\
& -\frac{G_{\Delta}}{\pi} \int \frac{d^{2} r}{a^{2}}: \sin \theta:: \cos \phi:-\frac{G_{c}}{\pi} \int \frac{d^{2} r}{a^{2}}: \cos 2 \theta: \\
& +\frac{G_{s}}{\pi} \int \frac{d^{2} r}{a^{2}}: \cos 2 \phi:-\frac{G_{c s}}{\pi} \int \frac{d^{2} r}{a^{2}}: \cos 2 \theta:: \cos 2 \phi: \\
& -\frac{G_{\rho s}}{\pi} \int d^{2} r\left(\partial_{z} \theta\right)\left(\partial_{z}^{-} \theta\right): \cos 2 \phi: \\
& +\frac{G_{c \sigma}}{\pi} \int d^{2} r\left(\partial_{z} \phi\right)\left(\partial_{z}^{-} \phi\right): \cos 2 \theta: \\
& +\frac{G_{\rho \sigma}}{\pi} \int d^{2} r a^{2}\left(\partial_{z} \theta\right)\left(\partial_{z}^{-} \theta\right)\left(\partial_{z} \phi\right)\left(\partial_{z}^{-} \phi\right)
\end{aligned}
$$

where $z=v_{F} \tau+i x, \quad \bar{z}=v_{F} \tau-i x, d^{2} r=v_{F} d x d \tau$, and $G_{i}$ $=g_{i} / 2 \pi v_{F}$. In this section, the operators are explicitly normal ordered.

In order to derive the $\mathrm{RG}$ equations, we use the following OPE's:

$$
\begin{gathered}
J_{\rho}(z) J_{\rho}(w)=\frac{1}{(z-w)^{2}}+\cdots, \\
\bar{J}_{\rho}(\bar{z}) \bar{J}_{\rho}(\bar{w})=\frac{1}{(\bar{z}-\bar{w})^{2}}+\cdots, \\
J_{\rho}(z): e^{i \alpha \theta(w, \bar{w})}:=\frac{\alpha}{2(z-w)}: e^{i \alpha \theta(w, \bar{w})}:+\cdots, \\
J_{\rho}(\bar{z}): e^{i \alpha \theta(w, \bar{w})}:=\frac{-\alpha}{2(\bar{z}-\bar{w})}: e^{i \alpha \theta(w, \bar{w})}:+\cdots,
\end{gathered}
$$




$$
\begin{aligned}
& : e^{i \alpha \theta(z, \bar{z})}:: e^{-i \alpha \theta(0,0)}: \\
& \quad=\frac{1}{|z|^{\alpha^{2}}}+\frac{\alpha}{|z|^{\alpha^{2}}}\left(z J_{\rho}-\bar{z} \bar{J}_{\rho}\right) \\
& \quad+\frac{2 i}{|z|^{2}}\left(\partial_{z} \partial_{z}^{-} \theta\right)+\frac{i \alpha}{2|z|^{\alpha^{2}}}\left[z^{2}\left(\partial_{z}^{2} \theta\right)+\bar{z}^{2}\left(\partial_{z}^{2} \theta\right)\right] \\
& \quad+\frac{\alpha^{2}}{2|z|^{\alpha^{2}}}\left[z^{2}: J_{\rho}^{2}:+\bar{z}^{2}: \bar{J}_{\rho}^{2}:\right]-\frac{\alpha^{2}}{|z|^{\alpha^{2}-2}} J_{\rho} \bar{J}_{\rho}+\cdots,
\end{aligned}
$$

$$
: e^{i \alpha \theta(z, \bar{z})}:: e^{i \beta \theta(0,0)}:=\frac{1}{|z|^{-\alpha \beta}}: e^{i(\alpha+\beta) \theta}:+\cdots,
$$

where we have introduced $\mathrm{U}(1)$ currents: $J_{\rho}(z) \equiv i \partial_{z} \theta(z, \bar{z})$, $\bar{J}_{\rho}(\bar{z}) \equiv-i \partial_{\bar{z}}^{-} \theta(z, \bar{z}), \quad J_{\sigma}(z) \equiv i \partial_{z} \phi(z, \bar{z}), \quad$ and $\quad \bar{J}_{\sigma}(\bar{z})$ $\equiv-i \partial_{\bar{z}}^{-} \phi(z, \bar{z})$. The parameters $\alpha$ and $\beta(\alpha+\beta \neq 0)$ in the vertex operator are numerical constants which determine the scaling dimension. In deriving the above OPE's, we have used the Wick theorem and the correlators: $\left\langle\theta_{+}(\bar{z}) \theta_{+}(\bar{\omega})\right\rangle$ $=-\frac{1}{2} \ln (\bar{z}-\bar{\omega}), \quad\left\langle\theta_{-}(z) \theta_{-}(\omega)\right\rangle=-\frac{1}{2} \ln (z-\omega), \quad$ and $\langle\theta(z, \bar{z}) \theta(\omega, \bar{\omega})\rangle=-\ln |z-\omega|$. From Eq. (B2), one finds

$$
\begin{gathered}
{\left[J_{\rho}(z) \bar{J}_{\rho}(\bar{z})\right]\left[J_{\rho}(0) \bar{J}_{\rho}(0)\right]} \\
=\frac{1}{|z|^{4}}+\frac{1}{|z|^{4}}\left(z^{2}: J_{\rho}^{2}:+\bar{z}^{2}: \bar{J}_{\rho}^{2}:\right)+\cdots, \\
{\left[J_{\rho}(z) \bar{J}_{\rho}(\bar{z})\right]: \cos \alpha \theta(0,0):=-\frac{\alpha^{2}}{4|z|^{2}}: \cos \alpha \theta:+\cdots,} \\
{\left[J_{\rho}(z) \bar{J}_{\rho}(\bar{z})\right]: \sin \alpha \theta(0,0):=-\frac{\alpha^{2}}{4|z|^{2}}: \sin \alpha \theta:+\cdots,}
\end{gathered}
$$

$: \cos \alpha \theta(z, \bar{z}):: \cos \alpha \theta(0,0):$

$$
\begin{aligned}
= & \frac{1}{2|z|^{\alpha^{2}}}+\frac{\alpha^{2}}{|z|^{\alpha^{2}}}\left(z^{2}: J_{\rho}^{2}:+\bar{z}^{2}: \bar{J}_{\rho}^{2}:\right) \\
& -\frac{\alpha^{2}}{2|z|^{\alpha^{2}-2}} J_{\rho} \bar{J}_{\rho}+\frac{1}{2}|z|^{\alpha^{2}}: \cos 2 \alpha \theta:+\cdots,
\end{aligned}
$$

$: \cos \alpha \theta(z, \bar{z}):: \cos \beta \theta(0,0):$

$$
\begin{aligned}
= & \frac{1}{2|z|^{\alpha \beta}}: \cos [(\alpha-\beta) \theta]: \\
& +\frac{1}{2|z|^{-\alpha \beta}}: \cos [(\alpha+\beta) \theta]:+\cdots .
\end{aligned}
$$

Exchanging $\theta \rightarrow \phi$ and $\rho \rightarrow \sigma$ yields the OPE's for spin phase fields.

Expanding the action in powers of coupling constants and integrating out short-distance parts, we obtain the scaling equations,

$$
\begin{gathered}
\frac{d}{d l} G_{\Delta}=G_{\Delta}\left(1+\frac{1}{2} G_{\rho}-\frac{1}{2} G_{\sigma}-G_{c}-G_{s}-\frac{1}{2} G_{c s}\right. \\
\left.-\frac{1}{4} G_{\rho s}-\frac{1}{4} G_{c \sigma}-\frac{1}{8} G_{\rho \sigma}\right) \\
\frac{d}{d l} G_{\rho}=+\frac{1}{4} G_{\Delta}^{2}+2 G_{c}^{2}+G_{c s}^{2}+G_{s} G_{\rho s} \\
\frac{d}{d l} G_{\sigma}=-\frac{1}{4} G_{\Delta}^{2}-2 G_{s}^{2}-G_{c s}^{2}-G_{c} G_{c \sigma}
\end{gathered}
$$

$$
\begin{aligned}
& \frac{d}{d l} G_{c}=-\frac{1}{4} G_{\Delta}^{2}+2 G_{\rho} G_{c}-\left(G_{s}+G_{\rho s}\right) G_{c s}, \\
& \frac{d}{d l} G_{s}=-\frac{1}{4} G_{\Delta}^{2}-2 G_{\sigma} G_{s}-\left(G_{c}+G_{c \sigma}\right) G_{c s},
\end{aligned}
$$

$$
\begin{aligned}
\frac{d}{d l} G_{c s}= & -\frac{1}{4} G_{\Delta}^{2}-2\left(1-G_{\rho}+G_{\sigma}+G_{\rho \sigma}\right) G_{c s} \\
& -2\left(G_{c}+G_{c \sigma}\right)\left(G_{s}+G_{\rho s}\right),
\end{aligned}
$$

$$
\begin{aligned}
\frac{d}{d l} G_{\rho s}= & -\frac{1}{4} G_{\Delta}^{2}-2\left(1+G_{\sigma}\right) G_{\rho s}+2 G_{\rho} G_{s} \\
& -4\left(G_{c}+G_{c \sigma}\right) G_{c s}-2 G_{s} G_{\rho \sigma},
\end{aligned}
$$

$$
\begin{aligned}
\frac{d}{d l} G_{c \sigma}= & -\frac{1}{4} G_{\Delta}^{2}-2\left(1-G_{\rho}\right) G_{c \sigma}-2 G_{\sigma} G_{c} \\
& -4\left(G_{s}+G_{\rho s}\right) G_{c s}-2 G_{c} G_{\rho \sigma},
\end{aligned}
$$

$$
\begin{aligned}
\frac{d}{d l} G_{\rho \sigma}= & -\frac{1}{4} G_{\Delta}^{2}-2 G_{\rho \sigma}+2 G_{\rho} G_{\sigma}-4 G_{c s}^{2} \\
& -4 G_{c} G_{c \sigma}-4 G_{s} G_{\rho s} .
\end{aligned}
$$

Here we note that the number of the RG equations can be reduced due to the spin-rotational $\mathrm{SU}(2)$ symmetry. To show this point more transparently, we introduce $X(l), Y(l)$, and $Z(l)$ by $X(l)=G_{\sigma}(l)-G_{s}(l), Y(l)=G_{c s}(l)-G_{c \sigma}(l)$, and $Z(l)=G_{\rho s}(l)-G_{\rho \sigma}(l)$. Their RG equations are obtained from Eqs. (B5)-(B12) as

$$
\frac{d}{d l} X=2 G_{s} X+\left(G_{c}-G_{c s}\right) Y
$$

$$
\frac{d}{d l} Y=2\left(-1+G_{\rho}+G_{s}+G_{\rho s}\right) Y+2\left(G_{c}-G_{c s}\right)(X-Z)
$$




$$
\frac{d}{d l} Z=-2\left(1-G_{s}\right) Z-2\left(G_{\rho}+G_{\rho s}\right) X-4\left(G_{c}-G_{c s}\right) Y .
$$

One immediately finds that, if $X(0)=Y(0)=Z(0)=0$, they vanish for all $l$, i.e., $X(l)=Y(l)=Z(l)=0$. This implies that $G_{\sigma}(l)=G_{s}(l), G_{c s}(l)=G_{c \sigma}(l)$, and $G_{\rho s}(l)=G_{\rho \sigma}(l)$, which are nothing but the constraints on the coupling constants due to the spin-rotational $\mathrm{SU}(2)$ symmetry. In this case, we can set $G_{\sigma}(l)=G_{s}(l), G_{c \sigma}(l)=G_{c s}(l)$, and $G_{\rho \sigma}(l)=G_{\rho s}(l)$ in the RG equations (B4)-(B12). Then the RG equations are given by Eqs. (4.12)-(4.17). The RG equations for the 1D EHM without the staggered site potential are obtained by setting $G_{\Delta}(l)=0$, Eqs. (2.20)-(2.24).

The RG equations can also be obtained in the presence of the bond dimerization in a similar way.
${ }^{1}$ For a review, see J.W. Bray, L.V. Interrante, I.S. Jacobs, and J.C. Bonner, in Extended Linear Chain Compounds, edited by J.S. Miller (Plenum, New York, 1983), Vol. 3, p. 353.

${ }^{2}$ T. Ishiguro, K. Yamaji, and G. Saito, Organic Superconductors (Springer-Verlag, Berlin, 1998).

${ }^{3}$ S. Kagoshima, R. Kato, H. Fukuyama, H. Seo, and H. Kino, in Advances in Synthetic Metals, Twenty Years of Progress in Science and Technology, edited by P. Bernier, S. Lefrant, and G. Bidan (Elsevier, New York, 1999), p. 262.

${ }^{4}$ M. Hase, I. Terasaki, and K. Uchinokura, Phys. Rev. Lett. 70, 3651 (1993).

${ }^{5}$ S. Sachdev, Physica A 313, 252 (2002).

${ }^{6}$ C.K. Majumdar and D.K. Ghosh, J. Math. Phys. 10, 1388 (1969); 10, 1399 (1969); C.K. Majumdar, J. Phys. C 3, 915 (1970); F.D.M. Haldane, Phys. Rev. B 25, 4925 (1982); K. Okamoto and K. Nomura, Phys. Lett. A 169, 433 (1992).

${ }^{7}$ I.I. Ukrainskiŭ, Zh. Eksp. Teor. Fiz. 76, 760 (1979) [ Sov. Phys. JETP 49, 381 (1979)].

${ }^{8}$ S. Kivelson and D.E. Heim, Phys. Rev. B 26, 4278 (1982).

${ }^{9}$ S. Mazumdar and S.N. Dixit, Phys. Rev. Lett. 51, 292 (1983); S.N. Dixit and S. Mazumdar, Phys. Rev. B 29, 1824 (1984).

${ }^{10}$ J.E. Hirsch, Phys. Rev. Lett. 51, 296 (1983).

${ }^{11}$ J. Hara, T. Nakano, and H. Fukuyama, J. Phys. Soc. Jpn. 51, 341 (1982); J. Hara and H. Fukuyama, ibid. 52, 2128 (1983).

${ }^{12}$ M. Sugiura and Y. Suzumura, J. Phys. Soc. Jpn. 71, 697 (2002).

${ }^{13}$ J. Málek, S.-L. Drechsler, S. Flach, E. Jeckelmann, and K. Kladko, J. Phys. Soc. Jpn. 72, 2277 (2003).

${ }^{14}$ P. Sengupta, A.W. Sandvik, and D.K. Campbell, Phys. Rev. B 67, 245103 (2003).

${ }^{15}$ M. Kuwabara, H. Seo, and M. Ogata, J. Phys. Soc. Jpn. 72, 225 (2003).

${ }^{16}$ M. Sugiura and Y. Suzumura, J. Phys. Soc. Jpn. 72, 1458 (2003).

${ }^{17}$ M. Nakamura, J. Phys. Soc. Jpn. 68, 3123 (1999); Phys. Rev. B 61, 16377 (2000); 65, 209902 (2002); M. Nakamura and J. Voit, ibid. 65, 153110 (2002).

${ }^{18}$ V.J. Emery, in Highly Conducting One-Dimensional Solids, edited by J. Devreese, R. Evrard, and V. van Doren (Plenum, New York, 1979), p. 247.

${ }^{19}$ R.A. Bari, Phys. Rev. B 3, 2662 (1971).

${ }^{20}$ J.E. Hirsch, Phys. Rev. Lett. 53, 2327 (1984).

${ }^{21}$ P.G.J. van Dongen, Phys. Rev. B 49, 7904 (1994).

${ }^{22}$ J.W. Cannon and E. Fradkin, Phys. Rev. B 41, 9435 (1990); J.W. Cannon, R.T. Scalettar, and E. Fradkin, ibid. 44, 5995 (1991).

${ }^{23}$ G.P. Zhang, Phys. Rev. B 56, 9189 (1997).

${ }^{24}$ J. Voit, Phys. Rev. B 45, 4027 (1992).

${ }^{25}$ P. Sengupta, A.W. Sandvik, and D.K. Campbell, Phys. Rev. B 65, 155113 (2002).
${ }^{26}$ See also A.W. Sandvik, P. Sengupta, and D.K. Campbell, Phys. Rev. Lett. 91, 089701 (2003). This is a comment to E. Jeckelmann, Phys. Rev. Lett. 89, 236401 (2002).

${ }^{27}$ N. Tomita and H. Fukutome, Solid State Commun. 81, 659 (1992); 81, 663 (1992).

${ }^{28}$ G.I. Japaridze, Phys. Lett. A 201, 239 (1995); G.I. Japaridze and A.P. Kampf, Phys. Rev. B 59, 12822 (1999); G.I. Japaridze and S. Sarkar, Eur. Phys. J. B 27, 139 (2002).

${ }^{29}$ H. Otsuka, Phys. Rev. Lett. 84, 5572 (2000); Phys. Rev. B 63, 125111 (2001).

${ }^{30}$ L. Arrachea, E.R. Gagliano, and A.A. Aligia, Phys. Rev. B 55, 1173 (1997); A.A. Aligia, K. Hallberg, C.D. Batista, and G. Ortiz, ibid. 61, 7883 (2000); M.E. Torio, A.A. Aligia, K. Hallberg, and H.A. Ceccatto, ibid. 62, 6991 (2000); M.E. Torio, A.A. Aligia, and H.A. Ceccatto, Phys. Rev. B 67, 165102 (2003).

${ }^{31}$ M. Nakamura and K. Itoh, J. Phys. Soc. Jpn. 70, 3606 (2001).

${ }^{32}$ M. Fabrizio, A.O. Gogolin, and A.A. Nersesyan, Phys. Rev. Lett. 83, 2014 (1999); Nucl. Phys. B 580, 647 (2000).

${ }^{33}$ M. Tsuchiizu and Y. Suzumura, J. Phys. Soc. Jpn. 68, 3966 (1999).

${ }^{34}$ Y. Takada and M. Kido, J. Phys. Soc. Jpn. 70, 21 (2001).

${ }^{35}$ J. Lou, S. Qin, T. Xiang, C. Chen, G.S. Tian, and Z. Su, Phys. Rev. B 68, 045110 (2003).

${ }^{36} \mathrm{Ph}$. Brune, G.I. Japaridze, A.P. Kampf, and M. Sekania, cond-mat/0106007 (unpublished); A.P. Kampf, M. Sekania, G.I. Japaridze, and Ph. Brune, J. Phys.: Condens. Matter 15, 5895 (2003).

${ }^{37}$ Y.Z. Zhang, C.Q. Wu, and H.Q. Lin, Phys. Rev. B 67, 205109 (2003).

${ }^{38}$ Y. Anusooya-Pati, Z.G. Soos, and A. Painelli, Phys. Rev. B 63, 205118 (2001).

${ }^{39}$ T. Wilkens and R.M. Martin, Phys. Rev. B 63, 235108 (2001).

${ }^{40}$ M.E. Torio, A.A. Aligia, and H.A. Ceccatto, Phys. Rev. B 64, 121105 (2001).

${ }^{41}$ M.C. Refolio, J.M. López Sancho, and J. Rubio, cond-mat/0210462 (unpublished).

${ }^{42}$ S. Caprara, M. Avignon, and O. Navarro, Phys. Rev. B 61, 15667 (2000).

${ }^{43}$ S. Gupta, S. Sil, and B. Bhattacharyya, Phys. Rev. B 63, 125113 (2001).

${ }^{44}$ K. Požgajčić and C. Gros, Phys. Rev. B 68, 085106 (2003).

${ }^{45}$ S.R. Manmana, V. Meden, R.M. Noack, and K. Schönhammer, cond-mat/0307741 (unpublished).

${ }^{46}$ M.J. Rice and E.J. Mele, Phys. Rev. Lett. 49, 1455 (1982).

${ }^{47}$ N. Nagaosa and J. Takimoto, J. Phys. Soc. Jpn. 55, 2735 (1986); 55, 2745 (1986); N. Nagaosa, ibid. 55, 2754 (1986).

${ }^{48}$ A. Girlando and A. Painelli, Phys. Rev. B 34, 2131 (1986); A. 
Painelli and A. Girlando, ibid. 37, 5748 (1988); 39, 9663 (1989).

${ }^{49}$ T. Egami, S. Ishihara, and M. Tachiki, Science 261, 1307 (1993); S. Ishihara, T. Egami, and M. Tachiki, Phys. Rev. B 49, 8944 (1994).

${ }^{50}$ R. Resta and S. Sorella, Phys. Rev. Lett. 74, 4738 (1995); 82, 370 (1999).

${ }^{51}$ N. Gidopoulos, S. Sorella, and E. Tosatti, Eur. Phys. J. B 14, 217 (2000).

${ }^{52}$ M. Tsuchiizu and A. Furusaki, Phys. Rev. Lett. 88, 056402 (2002).

${ }^{53}$ C. Nayak, Phys. Rev. B 62, 4880 (2000).

${ }^{54}$ J. Sólyom, Adv. Phys. 28, 201 (1979).

${ }^{55}$ K. Penc and F. Mila, Phys. Rev. B 50, 11429 (1994).

${ }^{56}$ J. Cardy, Scaling and Renormalization in Statistical Physics (Cambridge University Press, Cambridge, UK, 1996).

${ }^{57}$ A.O. Gogolin, A.A. Nersesyan, and A.M. Tsvelik, Bosonization and Strongly Correlated Systems (Cambridge University Press, Cambridge, 1998).

${ }^{58}$ T. Giamarchi and H.J. Schulz, Phys. Rev. B 39, 4620 (1989).

${ }^{59}$ Z. Bajnok, L. Palla, G. Takács, and F. Wágner, Nucl. Phys. B 601, 503 (2001).
${ }^{60}$ G. Delfino and G. Mussardo, Nucl. Phys. B 516, 675 (1998).

${ }^{61}$ M. Tsuchiizu, H. Yoshioka, and Y. Suzumura, J. Phys. Soc. Jpn. 70, 1460 (2001)

${ }^{62}$ K. Yonemitsu, Phys. Rev. B 65, 085105 (2002); 65, 205105 (2002).

${ }^{63}$ Brune et al. (Ref. 36) find very difficult to determine $U_{c 2}$ in their DMRG calculations, probably because a spin gap in the SDI phase becomes too small near $U=U_{c 2}$. Also, Wilkens and Martin (Ref. 39) did not find the SDW phase. This should be because their method of adding a finite dimerization $\delta$ and then taking the $\delta \rightarrow 0$ limit is delicate, as any small amount of $\delta$ is enough to change the SDW phase to the SDI phase (see Sec. V).

${ }^{64}$ W.P. Su, J.R. Schrieffer, and A.J. Heeger, Phys. Rev. Lett. 42, 1698 (1979); Phys. Rev. B 22, 2099 (1980).

${ }^{65}$ G. Ortiz, P. Ordejón, R.M. Martin, and G. Chiappe, Phys. Rev. B 54, 13515 (1996).

${ }^{66} \mathrm{R}$. Shankar, in Current Topics in Condensed Matter and Particle Physics, edited by J. Pati, Q. Shafi, and Y. Lu (World Scientific, Singapore, 1993), p. 83.

${ }^{67}$ J. von Delft and H. Schoeller, Ann. Phys. (Leipzig) 7, 225 (1998).

${ }^{68}$ I. Affleck, Nucl. Phys. B 265, 409 (1986). 